\title{
TWO DIMENSIONAL TOPOLOGY OPTIMIZATION OF HEAT EXCHANGERS WITH THE DENSITY AND LEVEL-SET METHODS.
}

\author{
SALAZAR DE TROYA, MIGUEL A. ${ }^{1}$; TORTORELLI, DANIEL A. ${ }^{2}$; BECK, VICTOR \\ A. ${ }^{3}$ \\ ${ }^{1}$ Lawrence Livermore National Laboratory \\ 7000 East Ave, Livermore, CA \\ salazardetro1@1lnl.gov \\ ${ }^{2}$ Lawrence Livermore National Laboratory \\ 7000 East Ave, Livermore, CA \\ tortorelli2@1lnl.gov \\ ${ }^{3}$ Lawrence Livermore National Laboratory \\ 7000 East Ave, Livermore, CA \\ beck33@1lnl.gov
}

Key words: Topology Optimization, Heat Exchanger, Stokes flow

\begin{abstract}
We design heat exchangers using two topology optimization approaches: the density, i.e. volume fraction and level set methods. Our goal is to maximize the heat exchange between two fluids in separate channels while constraining the pressure drop across each channel. The heat exchanger is modeled with a coupled thermal-flow formulation. The flow is governed by an isothermal and incompressible Stokes-Brinkman equation and the heat transfer is governed by a convection-diffusion equation with high Peclet number. We solve one set of Stokes-Brinkman equations per fluid. Each Brinkman term in the flow equation serves to model the other phase as a solid, thereby preventing mixing. We first represent the solid and fluid phases using a volume fraction variable and apply a SIMP-like penalization in the Brinkman term to drive the optimization to a discrete design. The cost and constraint function derivatives are automatically calculated with the library pyadjoint and the optimization is performed by the Method of Moving Asymptotes. In a second optimization formulation, we use the level set approach to define the interface that separates the two fluids. Pyadjoint calculates the shape derivatives of the cost and constraint functions and the Hamilton-Jacobi advects the interface, allowing for topological changes. We present results in two dimensions and discuss the advantages and disadvantages of each approach.
\end{abstract}

\section{Introduction}

Heat exchangers, that transfer heat between two or more fluids in separate channels, play an important role in a multitude of industries: power generation, chemical plants, automobiles, HVAC systems, etc. The main mechanism for heat transfer is conduction through the walls that separate the channels, therefore maximizing the channel surface area is key for performance. However, as the surface area increases so too does the pressure drop across the channels and hence the power required to operate the heat exchanger increases. 
Recent advances in manufacturing have permitted the miniaturization of heat exchangers. Greater effectiveness due to increased surface area to volume ratio, smaller thermal resistance and a larger range of applications to areas that require miniaturization, e.g. electronic circuits, biomedical processes, robotics, etc., cf. [81], [79]. High surface area to volume ratio comes nonetheless at the expense of a greater pressure drop across the flow channels which translates into a higher operating costs [40]. Designing a heat exchanger is therefore a compromise between heat transfer and pressure drop.

Most heat exchanger design studies are limited to a low-dimensional design space. The geometric configuration is typically fixed and a parametric optimization on channel cross sections or lengths is performed $[72,40,12,48]$. These design limitations come from manufacturing limitations. However, with the advent of additive manufacturing, these limitations can be relaxed. Engineers must, accordingly, develop new design tools to leverage this freedom.

One such design tool is topology optimization which finds the optimal geometry to minimize a given cost function and satisfy constraint functions. These functions depend on the solution of Partial Differential Equations (PDE) that model the physical phenomena of interest. The first applications of topology optimization were in structural mechanics. Notably, [19] formulated a material distribution problem wherein a volume fraction field models the solid, void and intermediate material phases. By penalizing the intermediate phase, e.g. by the SIMP (Solid Isotropic Material with Penalization) method, [91] designs with optimized hole placements and geometry are generated with distinct material and void phases. An alternative approach based on the level set method $[86,7,78]$ avoids the intermediate phase by using the zero isocontour of a level-set function to define the solid-void interface. However, the ability

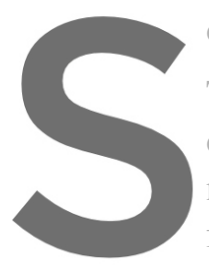
of introducing holes int

The aforementioned top optimization has subsequer netism [60], [92] and aco found in [37]. A more in-depth
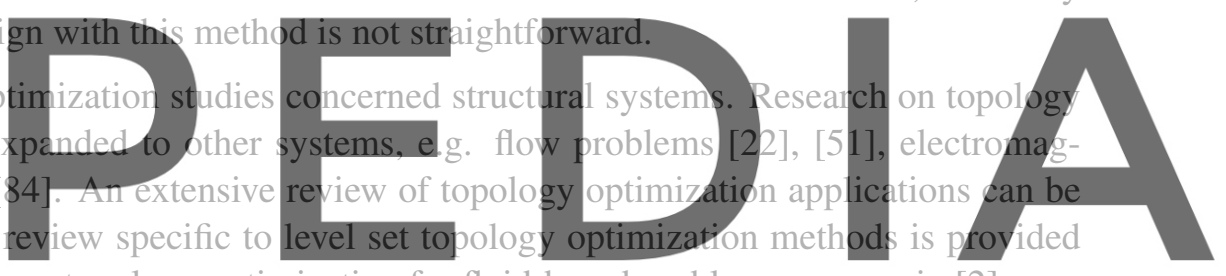

by [82] and a more recent review on topology optimization for fluid-based problems appears in [2].

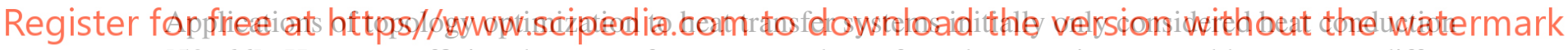
$[50,66]$. However, efficient heat transfer systems rely on forced convection, caused by pressure differences [38], [90], and natural convection, caused by buoyancy forces generated by temperature gradients in the fluid [1]. An extensive review of the design of heat transfer systems can be found in [35].

Few articles have been devoted to the design of heat exchangers and they often assume predetermined configurations of one flow channel [54], [61]. New methods are necessary to simultaneously optimize both the cooling and heating channel configurations. To the best of our knowledge, the first effort to do this is described in the [71] thesis, upon which this article is inspired. To keep the fluids in their separate channels, [71] solves two Stokes-Brinkman equations, one for the heating channel and one for the cooling channel. The material distribution of each channel is complementary to the other, e.g. the open channel region for the hot fluid channel is the solid region of the cold fluid channel. The walls separating the two fluids channels are assumed to be infinitesimally thin. [45] applies the level set method to heat exchanger design, wherein both channels are analyzed in one flow problem. A minimum distance constraint is imposed between the channels and thusly a finite wall thickness is obtained. The optimized results, however, use a predefined configuration. Therefore, the [45] method is shape optimization, rather than topology optimization. [76] obtains three-dimensional heat exchanger designs, however, very few details 
of the methodology are explained due to proprietary reasons. [54] design a two dimensional unit cell of an air-water heat exchanger. However, they do not simulate the water flow, rather the water temperature is prescribed as a boundary condition over the air-water interface. During the preparation of this work, two new articles on topology optimization of heat exchangers were published. [62] also uses [71] method as foundation and extends their work to three dimensions with the Navier-Stokes equation to model the flow. [58] also solves a three dimensional heat exchanger with the Navier-Stokes equation, although their designs feature walls with finite thickness by means of an erosion-dilation identification method.

Our work solves the exact same problem with the two most commonly used methods in topology optimization (the volume fraction and the level set methods) to highlight their differences. We also provide the source code used in this study for replication purposes and as a solid starting point for future researchers. More importantly, the codebase represents a methodology for the design of generalized two-phase systems that exchange species such as fuel cells, batteries, etc. In Section 2, we present the equations to model a two fluid heat exchanger, namely the Stokes-Brinkman and heat transfer equations. Section 3 discusses the numerical strategies to solve the topology optimization problem pertaining to the volume fraction and the level set methods. In Section 4, both methods are used to solve the same optimization problem: a parallel flow two dimensional heat exchanger. Finally, Section 5 discusses the conclusions and future research directions.

\section{Governing equations}

Heat exchangers are thermal fluid systems, where fluid mechanics and heat transfer interact with each

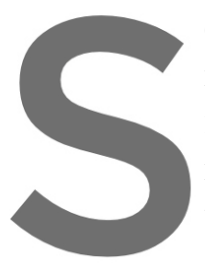
other in a highly complex incompressibility and low flow and neglect buoyan model their respective velocity pressure pair
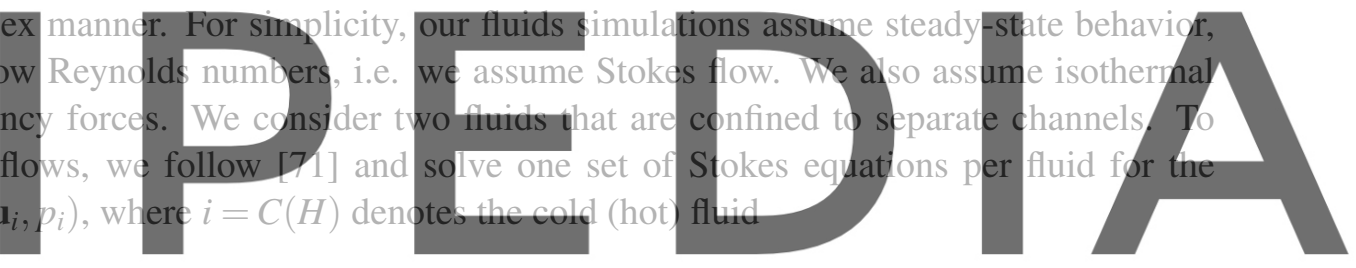

$$
-\mu \nabla^{2} \mathbf{u}_{i}+\nabla p_{i}=\mathbf{f}_{i} \quad \text { in } D_{i}
$$

Register for free at https//www.scipedia.Gomđo dpwnload the version without the watermark

$$
\begin{array}{ll}
\mathbf{u}_{i}=0 & \text { in } D \backslash D_{i}, \\
\mathbf{u}_{i}=\mathbf{u}_{i_{\text {in }}} & \text { on } \Gamma_{i_{\text {in }}}, \\
p_{i}=0 & \text { on } \Gamma_{i_{\text {out }}}, \\
\mathbf{u}_{i}=0 & \text { on } \partial D_{i} \backslash\left(\Gamma_{i_{\text {in }}} \cup \Gamma_{i_{\text {out }}}\right),
\end{array}
$$

where $\mu$ is the fluid viscosity. Note that we assume the same properties for the hot and cold fluids, e.g. $\mu=\mu_{C}=\mu_{H}$. The spatial domains $D_{C}$ and $D_{H}$ are complementary subsets of the total domain $D$, i.e. $D=D_{C} \cup D_{H}$. To avoid fluid mixing, we consider each complementary domain $D \backslash D_{i}$ as a solid phase with $\mathbf{u}_{i}=0$, e.g. $\mathbf{u}_{C}=0$ in $D_{H}=D \backslash D_{C}$ in the analysis of the cold fluid. This strategy assumes an infinitesimally thin interface separates the fluids. Fluid $i$ enters the chamber through surface $\Gamma_{i_{\text {in }}}$ with an imposed velocity $\mathbf{u}_{i_{\text {in }}}$ and exits the chamber through $\Gamma_{i_{\text {out }}}$ at the constant pressure $p_{i}=0$. The remaining boundary $\partial D_{i} \backslash \Gamma_{i_{\text {in }}} \cup \Gamma_{i_{\text {out }}}$ is subjected to the no-slip boundary condition.

Thermal energy is transported by advection and conduction and does not distinguish the phases, i.e. the 
temperature field $T$ is computed over the entire domain $D$ by solving

$$
\begin{aligned}
\rho c_{p} \nabla T \cdot\left(\mathbf{u}_{C}+\mathbf{u}_{H}\right)+k \nabla^{2} T & =0 & & \text { in } D, \\
T & =\hat{T}_{C} & & \text { on } \Gamma_{C_{\text {in }}}, \\
T & =\hat{T}_{H} & & \text { on } \Gamma_{H_{\text {in }}}, \\
\nabla T \cdot \mathbf{n} & =0 & & \text { on } \Gamma \backslash\left(\Gamma_{H_{\text {in }}} \cup \Gamma_{C_{\text {in }}} \cup \Gamma_{H_{\text {out }}} \cup \Gamma_{C_{\text {out }}}\right),
\end{aligned}
$$

where $c_{p}$ is the specific heat capacity at constant pressure, $\rho$ is the fluid density and $k$ is the conduction coefficient. Again, note that we assume the same properties for the hot and cold fluids. The flow velocities $\mathbf{u}_{C}$ and $\mathbf{u}_{H}$ give rise to the advection energy transport. Adiabatic boundary conditions are enforced over all surfaces with the exception of the channel inlets and outlets.

The cold and hot fluid domains, $D_{C}$ and $D_{H}$ respectively, evolve during the optimization and hence their geometries are not known a priori. To model their domains we follow an Ersatz approach and simulate the solid phases $D \backslash D_{i}$ via a Brinkman penalization term $\alpha \chi_{i}(\mathbf{x}) \mathbf{u}_{i}$ [22] where $\alpha$ is a sufficiently high scalar and $\chi_{i}: D \rightarrow\{0,1\}$ are the indicator functions defined such that

$$
\chi_{C}(\mathbf{x})=\left\{\begin{array}{l}
0 \text { in } D_{C} \\
1 \text { else }
\end{array}\right.
$$

and

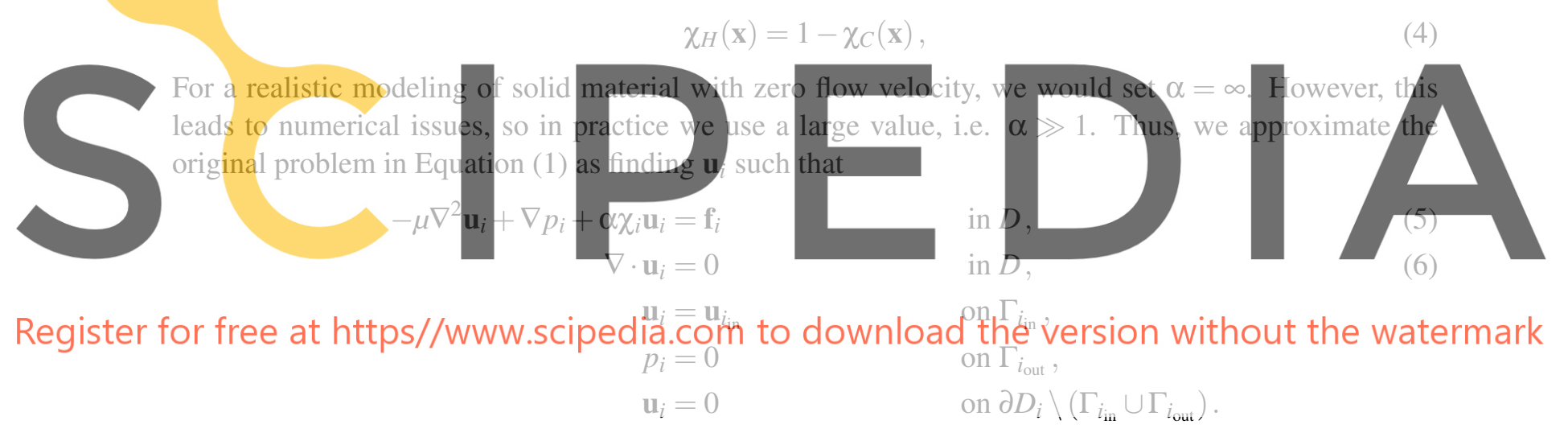

We discretize the Stokes equation with the finite element method over the triangular partition $\mathcal{T}_{h}$ of $D$. We multiply Equations (5) and (6) with the test functions $\mathbf{v}_{i}$ and $q_{i}$, integrate over the domain $D$ and apply integration by parts to obtain the weak formulation: Find $\left(\mathbf{u}_{i}, p_{i}\right) \in \mathbf{V}_{i}\left(\mathcal{T}_{h}\right) \times Q_{i}\left(\mathcal{T}_{h}\right)$ such that

$$
\begin{aligned}
a\left(\chi_{i} ; \mathbf{u}_{i}, \mathbf{v}_{i}\right)+b\left(p_{i}, \mathbf{v}_{i}\right) & =l\left(\mathbf{v}_{i}\right), \\
b\left(q_{i}, \mathbf{u}_{i}\right) & =0,
\end{aligned}
$$

for all test functions $\left(\mathbf{v}_{i}, q_{i}\right) \in \mathbf{V}_{i}\left(\mathcal{T}_{h}\right) \times Q_{i}\left(\mathcal{T}_{h}\right)$ and $i=C, H$, where

$$
\begin{aligned}
a\left(\chi_{i} ; \mathbf{u}_{i}, \mathbf{v}_{i}\right) & =\int_{D} \mu \nabla \mathbf{u}_{i}: \nabla \mathbf{v}_{i} d V+\int_{D} \alpha \chi_{i} \mathbf{u}_{i} \cdot \mathbf{v}_{i} d V, \\
b\left(p_{i}, \mathbf{v}_{i}\right) & =\int_{D} p_{i} \nabla \cdot \mathbf{v}_{i} d V \\
l\left(\mathbf{v}_{i}\right) & =\int_{D} \mathbf{f}_{i} \cdot \mathbf{v}_{i} d V
\end{aligned}
$$


with the finite element function spaces

$$
\begin{aligned}
\mathbf{V}_{i}\left(\mathcal{T}_{h}\right) & =\left\{\mathbf{v} \in\left[C^{0}(D)\right]^{d}:\left.\mathbf{v}_{h}\right|_{\mathcal{K}} \in\left[P^{2}(\mathcal{K})\right]^{d} \forall \mathcal{K} \in \mathcal{T}_{h} \mid \mathbf{v}=\mathbf{u}_{i_{\text {in }}} \text { on } \Gamma_{i_{\text {in }}}\right\}, \\
\mathbf{V}_{0_{i}}\left(\mathcal{T}_{h}\right) & =\left\{\mathbf{v} \in\left[C^{0}(D)\right]^{d}:\left.\mathbf{v}_{h}\right|_{\mathcal{K}} \in\left[P^{2}(\mathcal{K})\right]^{d} \forall \mathcal{K} \in \mathcal{T}_{h} \mid \mathbf{v}=\mathbf{0} \text { on } \Gamma_{i_{\text {in }}}\right\}, \\
Q_{i}\left(\mathcal{T}_{h}\right) & =\left\{q \in C^{0}(D):\left.q_{h}\right|_{\mathcal{K}} \in P^{1}(\mathcal{K}) \forall \mathcal{K} \in \mathcal{T}_{h} \mid q=0 \text { on } \Gamma_{i_{\text {out }}}\right\},
\end{aligned}
$$

where $d$ is the mesh geometric dimension. This pair of finite element spaces $\mathbf{V}_{i}\left(\mathcal{T}_{h}\right)$ and $Q_{i}\left(\mathcal{T}_{h}\right)$ are synonymous with the Taylor-Hood element pair of lowest order (second order Lagrange elements for the velocity and first order Lagrange elements for the pressure) and satisfy the Ladyzhenskaya-BabuskaBrezzi (LBB) conditions [24]. For conciseness, we henceforth refer to $\mathbf{V}_{i}\left(\mathcal{T}_{h}\right), \mathbf{V}_{0_{i}}\left(\mathcal{T}_{h}\right)$ and $Q_{i}\left(\mathcal{T}_{h}\right)$ as $\mathbf{V}_{i}$, $\mathbf{V}_{0_{i}}$ and $Q_{i}$.

Equation (2) is dominated by convection and it is well known that the standard Galerkin finite element method does not converge for convection dominated problems [25]. As such, we use the Discontinuous Galerkin method to solve Equation (2). The convective component is accommodated with an upwind scheme and the elliptic component is discretized with the symmetric interior penalty Galerkin formulation (SIPG). We apply both the upwind scheme and the SIPG to Equation (2) to obtain the weak form: Find $T \in W\left(\mathcal{T}_{h}\right)=\left\{v_{h} \in L^{2}(D):\left.v_{h}\right|_{\mathcal{K}} \in P^{1}(\mathcal{K}) \forall \mathcal{K} \in \mathcal{T}_{h}\right\}$ such that

$$
a_{T}(\mathbf{u} ; T, w)=l_{T}(w),
$$

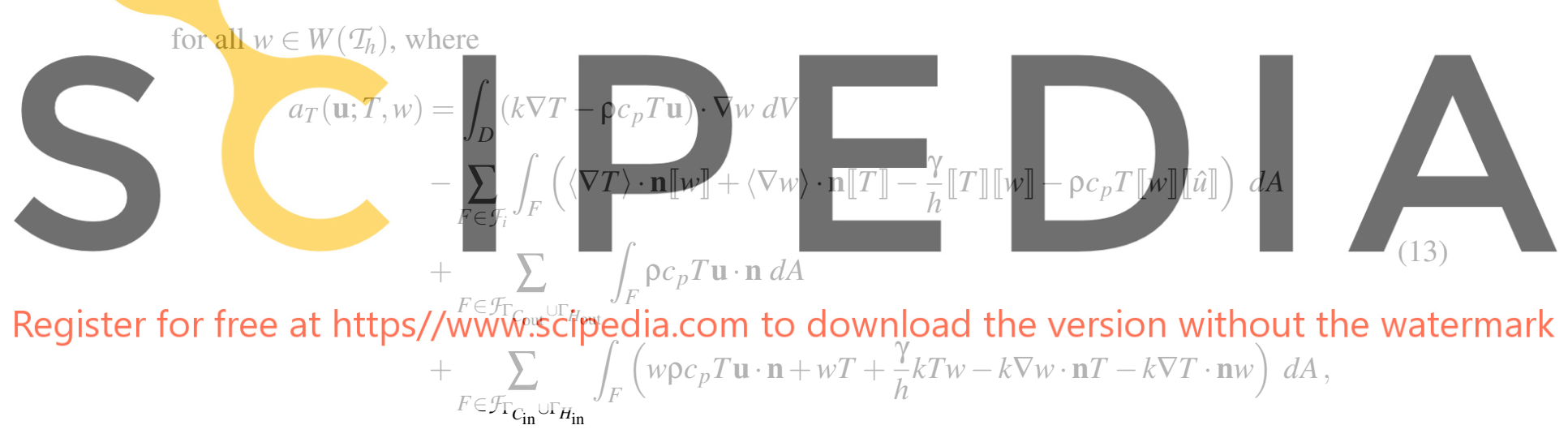

and

$$
l_{T}(w)=\sum_{F \in \mathcal{F}_{\Gamma_{\mathrm{in}}}} \int_{F}\left(\hat{T}_{C} k \nabla w \cdot \mathbf{n}+w \hat{T}_{C}+\frac{\gamma}{h} \hat{T}_{C} w\right) d A+\sum_{F \in \mathcal{F}_{\Gamma_{H_{\mathrm{in}}}}} \int_{F}\left(\hat{T}_{H} k \nabla w \cdot \mathbf{n}+w \hat{T}_{H}+\frac{\gamma}{h} \hat{T}_{H} w\right) d A,
$$

in which $\mathbf{u}=\mathbf{u}_{C}+\mathbf{u}_{H}$,

$$
\hat{u}=\mathbf{u} \cdot \mathbf{n}+\left|\frac{\mathbf{u} \cdot \mathbf{n}}{2}\right|,
$$

is the upwind flux, $\gamma$ is a penalty parameter equal to $5 \times 10^{4}$ herein, $h$ is the cell diameter and $\mathbf{n}$ is the element face $F$ normal vector. The integration set $\mathcal{F}_{i}$ is comprised of all interior element faces, whereas $\mathcal{F}_{\Gamma}, \mathcal{F}_{\Gamma_{C_{\text {in }}}}, \mathcal{F}_{\Gamma_{H_{\mathrm{in}}}}, \mathcal{F}_{\Gamma_{C_{\text {out }}}}$ and $\mathcal{F}_{\Gamma_{H_{\text {out }}}}$ are comprised of the element faces on the boundaries $\Gamma, \Gamma_{C_{\text {in }}}, \Gamma_{H_{\text {in }}}, \Gamma_{C_{\text {out }}}$ and $\Gamma_{H_{\text {out }}}$ respectively. Note that the Dirichlet boundary conditions, cf. Equations (2b), (2c), are weakly enforced. 


\section{Topology optimization of heat exchangers}

Heat exchangers are designed to maximize energy transfer. As such, we formulate the topology optimization problem in terms of our design field, i.e. the cold fluid domain characteristic function (from which the hot fluid domain is defined, cf. Equation (4)) $\chi_{C} \in X=\{f: D \rightarrow\{0,1\}\}$ such that

$$
\max _{\chi_{C} \in X} J\left(\chi_{C}\right)=\int_{\Gamma_{C_{\text {out }}}} T \rho c_{p} \mathbf{u}_{C} \cdot \mathbf{n} d A,
$$

s.t. $\left(\mathbf{u}_{C}, \mathbf{u}_{H}, p_{C}, p_{H}, T\right) \in \mathbf{V}_{C} \times \mathbf{V}_{H} \times Q_{C} \times Q_{H} \times W$ satisfy

$$
\begin{aligned}
a\left(\chi_{C} ; \mathbf{u}_{C}, \mathbf{v}_{C}\right)+b\left(\mathbf{v}_{C}, p_{C}\right)+b\left(\mathbf{u}_{C}, q_{C}\right) & =l\left(\mathbf{v}_{C}\right), \\
a\left(\chi_{H} ; \mathbf{u}_{H}, \mathbf{v}_{H}\right)+b\left(\mathbf{v}_{H}, p_{H}\right)+b\left(\mathbf{u}_{H}, q_{H}\right) & =l\left(\mathbf{v}_{H}\right), \\
a_{T}\left(\mathbf{u}_{C}+\mathbf{u}_{H} ; T, w\right) & =l_{T}(w),
\end{aligned}
$$

for all $\left(\mathbf{v}_{C}, \mathbf{v}_{H}, q_{C}, q_{H}, w\right) \in \mathbf{V}_{0_{C}} \times \mathbf{V}_{0_{H}} \times Q_{C} \times Q_{H} \times W$

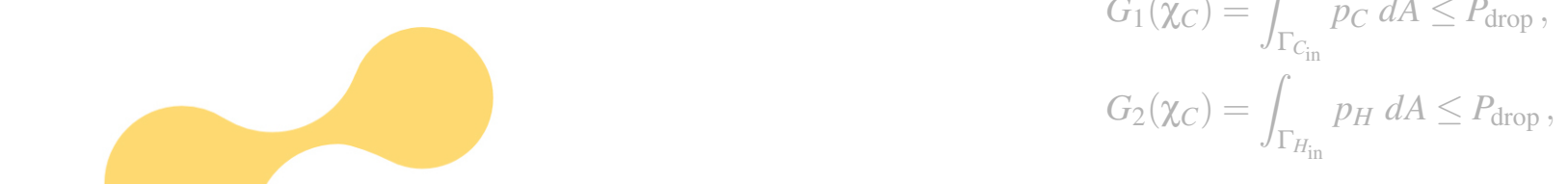

$$
\begin{aligned}
& G_{1}\left(\chi_{C}\right)=\int_{\Gamma_{\mathrm{in}}} p_{C} d A \leq P_{\text {drop }}, \\
& G_{2}\left(\chi_{C}\right)=\int_{\Gamma_{H_{\mathrm{in}}}} p_{H} d A \leq P_{\text {drop }},
\end{aligned}
$$

i.e. our goal is to maximize the energy flux through the cold fluid outlet $\Gamma_{C_{\text {out }}}$. To prevent an excessive power requirement, the constraints $G_{1}\left(\chi_{C}\right)$ and $G_{2}\left(\chi_{C}\right)$ limit the pressure drop across each fluid channel to be less than $P_{\text {drop. }}$. This constraint also serves to regularize the problem. Indeed, withont a pressure constraint, the optimal areas to maximize the inte drop. Our optimization problem (16) is not tractable for n the characteristic function yields a discrete optimiz

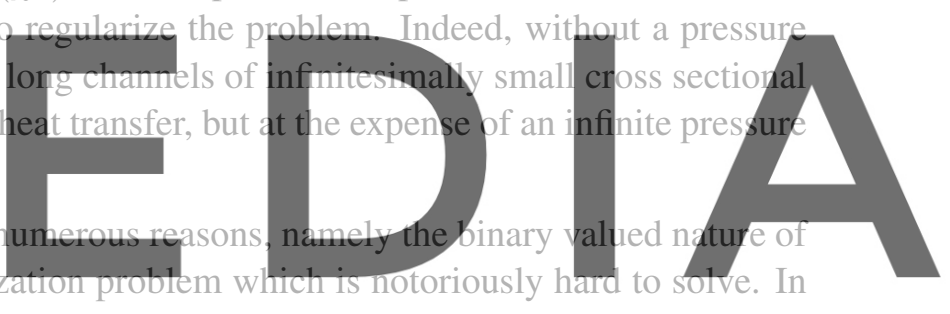

the following paragraphs we devise two strategies to transform this problem into ones that are solvable

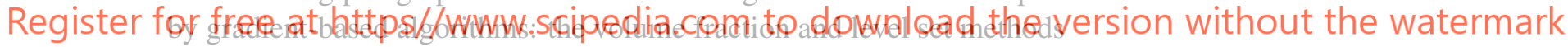

\subsection{Volume fraction method}

The so called volume-fraction method (also known as density or material distribution method) consists of replacing the binary-valued design field $\chi_{C}: D \rightarrow\{0,1\}$ with the convex volume fraction (of cold versus hot material) field $v_{C}: D \rightarrow[0,1]$ and penalizing intermediate values so that $v_{C}$ best resembles the indicator function $\chi_{C}$. For this purpose, we use the SIMP formulation [20] and define the penalized volume fraction as

$$
\hat{\mathrm{v}}(\mathrm{v})=\mathrm{v}^{p_{\text {SIMP }}}
$$

where $p_{\text {SIMP }}$ is a penalization parameter. SIMP originated in structural optimization where there is a natural trade-off between stiffness and weight. Here, a similar trade-off exists between heat transfer and pressure drop. 
Replacing $\chi_{C}$ for $v_{C}$ penalized by SIMP, cf. Equation (17), in Equation (16), we obtain

$$
\max _{v_{C} \in V} J\left(v_{C}\right)=\int_{\Gamma_{C_{\text {out }}}} T \rho c_{p} \mathbf{u}_{C} \cdot \mathbf{n} d A,
$$

s.t. $\left(\mathbf{u}_{C}, \mathbf{u}_{H}, p_{C}, p_{H}, T\right) \in \mathbf{V}_{C} \times \mathbf{V}_{H} \times Q_{C} \times Q_{H} \times W$ satisfy

$$
\begin{aligned}
a\left(\hat{\mathbf{v}}\left(\mathrm{v}_{C}\right) ; \mathbf{u}_{C}, \mathbf{v}_{C}\right)+b\left(\mathbf{v}_{C}, p_{C}\right)+b\left(\mathbf{u}_{C}, q_{C}\right) & =l\left(\mathbf{v}_{C}\right), \\
a\left(\hat{\mathbf{v}}\left(\mathrm{v}_{H}\right) ; \mathbf{u}_{H}, \mathbf{v}_{H}\right)+b\left(\mathbf{v}_{H}, p_{H}\right)+b\left(\mathbf{u}_{H}, q_{H}\right) & =l\left(\mathbf{v}_{H}\right), \\
a_{T}\left(\mathbf{u}_{C}+\mathbf{u}_{H} ; T, w\right) & =l_{T}(w),
\end{aligned}
$$

for all $\left(\mathbf{v}_{C}, \mathbf{v}_{H}, q_{C}, q_{H}, w\right) \in \mathbf{V}_{0_{C}} \times \mathbf{V}_{0_{H}} \times Q_{C} \times Q_{H} \times W$,

$$
\begin{aligned}
& G_{1}\left(v_{C}\right)=\int_{\Gamma_{C_{\mathrm{in}}}} p_{C} d A \leq P_{\mathrm{drop}}, \\
& G_{2}\left(\mathrm{v}_{C}\right)=\int_{\Gamma_{H_{\mathrm{in}}}} p_{H} d A \leq P_{\mathrm{drop}},
\end{aligned}
$$

where we note $v_{H}=1-v_{C}$. The function space $V=\left\{v \in L^{2}(D) \mid 0 \leq v \leq 1\right\}$ is discretized using element-wise uniform values.

We use a reduced space optimization formulation to solve Problem (18) and calculate the cost and constraint functions $J\left(v_{C}\right), G_{1}\left(v_{C}\right)$ and $G_{2}\left(v_{C}\right)$ and their sensitivities $D J\left(v_{C}\right), D G_{1}\left(v_{C}\right)$ and $D G_{2}\left(v_{C}\right)$ using Firedrake $[75,67,57]$ and the adjoint method with pyadjoint [68]. For the sensitivity computations, we consider the dependency of $\mathbf{u}_{C}, p_{C}, \mathbf{u}_{H}, p_{H}$ and $T$ on the design $v_{C}$ so that, e.g., $J\left(v_{C}\right)=\hat{J}\left(v_{C}, \mathbf{u}_{C}\left(v_{C}\right)\right.$. $p_{C}\left(\mathrm{v}_{C}\right), \mathbf{u}_{H}\left(\mathrm{v}_{C}\right), p_{H}\left(\mathrm{v}_{C}\right)$ the MMA algorithm [80] tolerance is satisfied.

3.2 Level set method
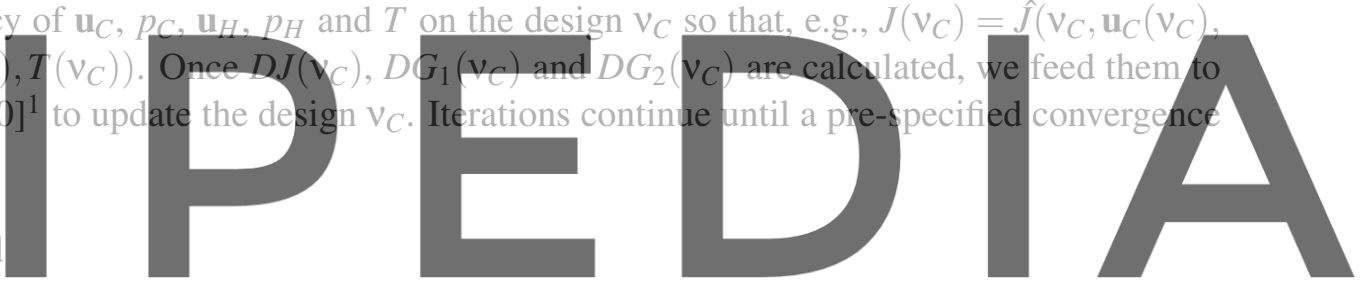

The level set method [70] is a numerical technique to describe the evolution of an interface as the zero

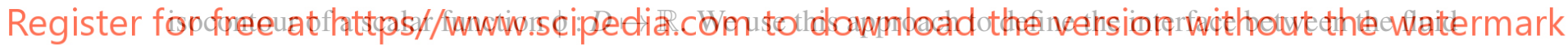
channels, which we denote as $\partial D_{C}$ such that

$$
\begin{aligned}
& \phi(\mathbf{x})>0, \mathbf{x} \in D_{H} \text { Hot fluid } \\
& \phi(\mathbf{x})=0, \mathbf{x} \in \partial D_{C} \text { Interface, } \\
& \phi(\mathbf{x})<0, \mathbf{x} \in D_{C} \text { Cold fluid }
\end{aligned}
$$

In each optimization iteration, the shape derivative with respect to $D_{C}$ is used to advect the $\phi(\mathbf{x})=0$ isocontour. As such, it is possible to merge existing holes, but not nucleate new holes.

The level-set function $\phi$ is discretized over a regular mesh via linear interpolation, i.e. Lagrange shape functions. Because of this, the material interface $\partial D_{C}$ is rarely aligned with the mesh elements. This misalignment compromises the accuracy of the forward problem (8). This well known problem is resolved by using conformal meshes that adhere to the interface $[4,89]$, applying immersed boundary techniques $[31,83,18,63]$ and by implementing an Ersatz material approach [8]. For more details on these three approaches, we refer to [82].

\footnotetext{
${ }^{1}$ More specifically, we use an adapted MMA python implementation from the GetDP finite element library [42].
} 
In this work, we implement the Ersatz method. We proceed by replacing the indicator function $\chi_{C}$ in Equation (8) with $H \circ \phi: D \rightarrow \mathbb{R}$ where $H$ is the unit step function, whereupon, e.g. $\int_{D \backslash D_{C}} f(x) d V=$ $\int_{D} f(x) H(\phi(x)) d V$. In the finite element discretization, the function $H \circ \phi$ is interpolated with linear Lagrange elements. Therefore, in elements where the level set $\phi$ changes sign, the effective penalization coefficient $\alpha \cdot(H \circ \phi)$ varies linearly between $\alpha$ and zero. As such we have a blurred interphase region rather than a sharp interface between the hot and cold fluid channels. Fortunately, the size of the interphase region can be reduced through mesh refinement. The lack of differentiability of $H$ is only a concern in the unlikely scenario when $\phi(\mathbf{x})=0$ at the element nodes. If, e.g. some of the level set values of an element's nodes are negative and the others equal zero then the design will be non-differentiable. Indeed, a "positive" design change to a zero level set valued node will move the entire element into the cold fluid domain whereas a "negative" change will keep the entire element in the hot domain. The effect of this anomaly is also lessened with mesh refinement.

The optimization problem in Equation (16) is now formulated as a shape optimization problem. Letting $O$ denote the set of all admissible domains:

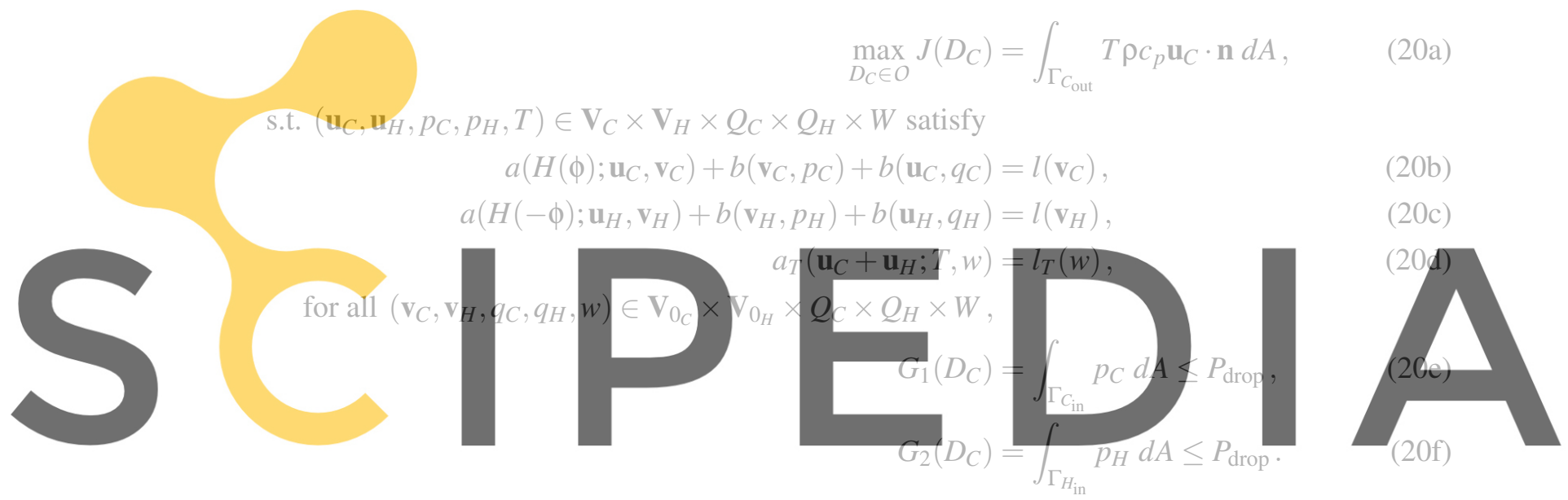

Register for free at https//www.scipedia.com to download the version without the watermark domain $D_{C}$ since the hot fluid domain $D_{H}=D \backslash D_{C}$. In the above, we remind the reader that $\phi$ and $D_{C}$ define the same domain, cf. Equation (19).

\subsubsection{Shape derivatives}

In the level set method literature, the shape sensitivities are calculated by either differentiating the continuum equations and then discretizing the resulting expressions for computational purposes or vice versa, i.e. discretizing the PDEs and cost and constraint functions and then differentiating them. Other terms for the same concept are differentiate-discretize and discretize-differentiate approaches respectively. Our choice is to calculate the shape derivatives $D J\left(D_{C}\right), D G_{1}\left(D_{C}\right)$ and $D G_{2}\left(D_{C}\right)$ using the differentiate-discretize approach. This choice allows us to take advantage of the automatic shape derivative calculation in the Unified Form Language (UFL) [55] within the pyadjoint library [41], [68]. As in the volume-fraction method, we also use a reduced space formulation and hence we consider the dependency of the primal variables $\mathbf{u}_{C}, p_{C}, \mathbf{u}_{H}, p_{H}$ and $T$ on the design $D_{C}$ so that, e.g. $J\left(D_{C}\right)=$ $\hat{J}\left(D_{C}, \mathbf{u}_{C}\left(D_{C}\right), p_{C}\left(D_{C}\right), \mathbf{u}_{H}\left(D_{C}\right), p_{H}\left(D_{C}\right), T\left(D_{C}\right)\right)$. 
Following [64], a general functional $J\left(D_{C}\right)$ evaluated on the design $D_{C}$ admits a shape derivative $D J\left(D_{C}\right)[\xi]$ in the direction $\xi \in \Psi(D)=\left\{\xi \in C^{s}\left(\mathbb{R}^{d}\right) \mid \xi \cdot \mathbf{n}_{\mid \partial D}=0\right\}$ (with integer $s \geq 0$ ) in the form

$$
D J\left(D_{C}\right)[\xi]=\int_{D}(\Pi: \nabla \xi+\mathfrak{\imath} \cdot \xi) d V
$$

where $\Pi$ and $\imath$ are sufficiently smooth tensor and vector fields. Given enough regularity assumptions, the Hadamard-Zolésio theorem [39] shows that Equation (21) only depends on the restriction to the boundary

$$
D J\left(D_{C}\right)[\xi]=\int_{\partial D_{C}} g \xi \cdot n d A
$$

where $g:=\left[\left(\left.\Pi\right|_{D_{C}}-\left.\Pi\right|_{D \backslash D_{C}}\right) n\right] \cdot n$. We opt however, to use the Equation (21) option because it is easier to automatically calculate with pyadjoint, as surface integrals in our Ersatz approach are difficult to evaluate. Moreover, it also offers better accuracy $[33,56]$, and it is identical to the discretize and then differentiate approach [21].

\subsubsection{Velocity extension and regularization}

The domain shape derivative $D J\left(D_{C}\right)[\xi]$ of Equation (21) requires the specification of a shape velocity field $\zeta$ throughout $D$, rather than just over the boundary $\partial D$ as required by the Equation (22) expression. This domain specification is known as the extension problem [82]. Here, we follow [36] and use a Hilbertian extension which helps to accelerate convergence when using the Ersatz approach, regular-

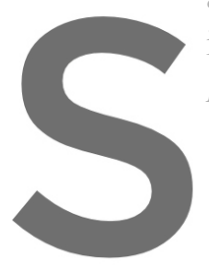
izes the shape derivativ $D J\left(D_{C}\right)[\xi]$, we proceed
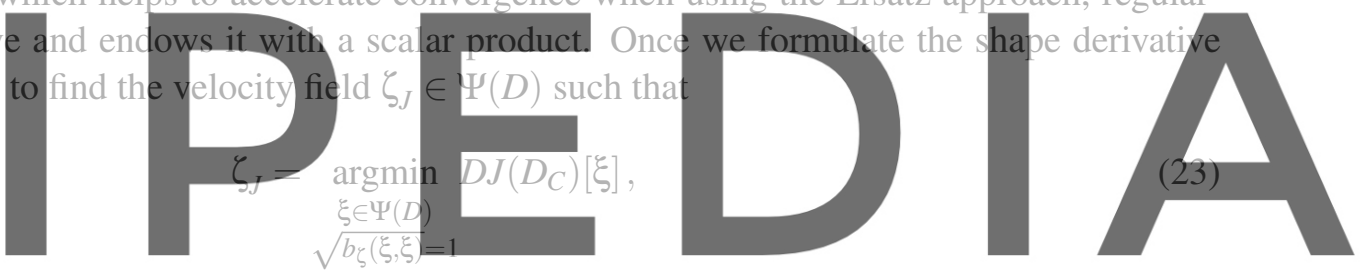

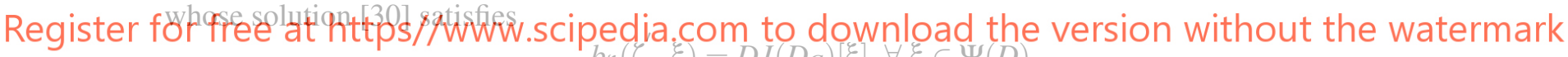
$\left.b_{\zeta}\left(\zeta_{J}, \xi\right)=D J\left(D_{C}\right) \xi \xi\right] \forall \xi \in \Psi(D)$

$$
\zeta_{J}=\frac{\zeta_{J}^{\prime}}{\sqrt{b_{\zeta}\left(\zeta_{J}^{\prime}, \zeta_{J}^{\prime}\right)}}
$$

where

$$
b_{\zeta}(\zeta, \xi)=\int_{D} \gamma \nabla \zeta: \nabla \xi d V+\int_{D} \zeta \cdot \xi d V+c_{1} \int_{\partial D}(\zeta \cdot \mathbf{n})(\xi \cdot \mathbf{n}) d A .
$$

The parameter $\gamma$ regularizes the problem and helps to accelerate convergence [36, 27]. It must be set large enough so that $\zeta$ extends beyond the boundary $\partial D_{C}$ region, but small enough that values of $\zeta$ in one boundary subregion do not greatly affect the other boundary subregions. The boundary integral on $\partial D$ weighted by the parameter $c_{1} \gg 1$ helps to enforce a zero normal component of the velocity $\zeta$ on the boundary $\partial D$. The same regularization is performed on $D G_{1}\left(D_{C}\right)$ and $D G_{2}\left(D_{C}\right)$ to obtain $\zeta_{G_{1}}$ and $\zeta_{G_{2}}$. 


\subsubsection{Optimization algorithm}

Once $\zeta_{J}, \zeta_{G_{1}}$ and $\zeta_{G_{2}}$ are calculated, we use them to calculate a search direction that decreases the cost function and accommodates the constraint functions $G_{1}\left(D_{C}\right)$ and $G_{2}\left(D_{C}\right)$ in Problem (20). Some researchers minimize a Lagrangian obtained by adding the constraint functions weighted by a crude estimation of the Lagrange multipliers to the cost function. This approach does not ensure satisfaction of the constraints, but it is sometimes sufficient to obtain reasonable designs. Other approaches use the Augmented Lagrange method [3], the sequential linear programming method [44] and the null space gradient flow algorithm [47]. In this work, we opt for the latter algorithm due to the easy availability as open source code in python [46]. The null space algorithm calculates a search direction $\theta \in \Psi(D)$ using the cost function and constraint functions gradients obtained with Equation (24). For more details, we refer to [47].

\subsubsection{Level set evolution and reinitialization}

The scalar function $\phi_{n}: D \rightarrow \mathbb{R}$ at the optimization iteration $n$ is advected in the search direction $\theta$ by taking $m$ time steps with time step $d t$ of the Hamilton-Jacobi equation

$$
\begin{aligned}
\frac{\partial \phi}{\partial t} & =\theta \cdot \nabla \phi \quad 0 \leq t \leq m d t, \\
(\mathbf{x}, 0) & =\phi_{n}(\mathbf{x}) .
\end{aligned}
$$

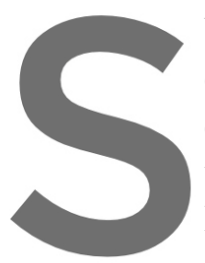

We represent the solutio

on the time step $d t$ using

decrease criteria merit

until satisfaction of the

Details on the numerical
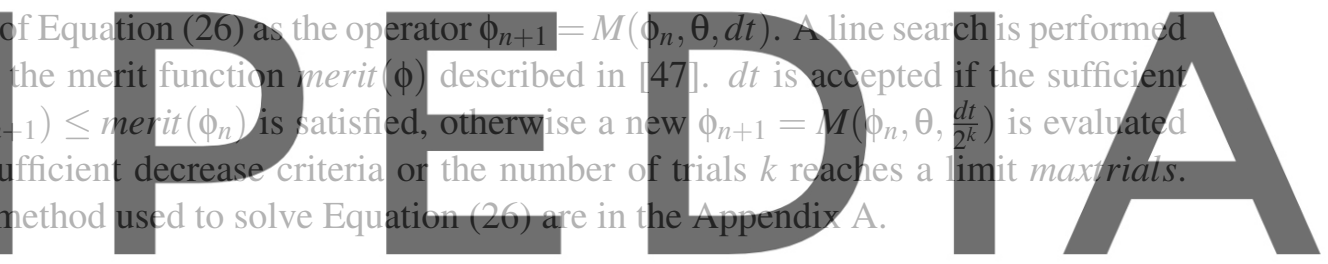

We apply a reinitialization procedure on $\phi_{n}$ every five optimization iterations to prevent it from becoming

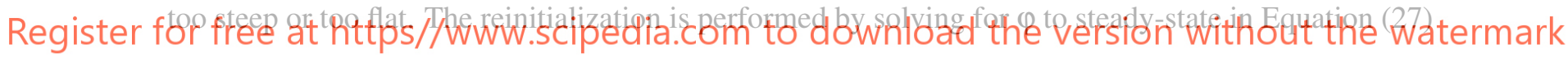

$$
\begin{aligned}
\frac{\partial \varphi}{\partial \tau}+\frac{\phi_{n}}{\sqrt{\phi_{n}^{2}+\varepsilon\left|\nabla \phi_{n}\right|^{2}}}(1-|\nabla \varphi|) & =0, \\
\varphi(\mathbf{x}, 0) & =\phi_{n}(\mathbf{x}),
\end{aligned}
$$

as presented by [73]. In the above, $\tau$ is a pseudo-time variable, $\phi_{n}$ is the level set function we intend to reinitialize and $\varepsilon$ is a stabilization parameter related to the mesh size. In practice, the reinitialization is not performed to steady-state but only few time steps are taken. Once $\varphi$ is obtained, we update $\phi_{n}=\varphi$ and proceed to the next optimization iteration. We describe the numerical procedure used to solve Equation (27) in Appendix B.

The optimization procedure is summarized in Algorithm 1.

\section{Numerical Examples}

Our simulation domain is similar to the one found in [71], cf. Figure 1. The cold and hot fluids of equal viscosity $\mu$ enter the chamber from the left at the inlets $\Gamma_{C_{\text {in }}}$ and $\Gamma_{H_{\text {in }}}$ and exit through the right at the 


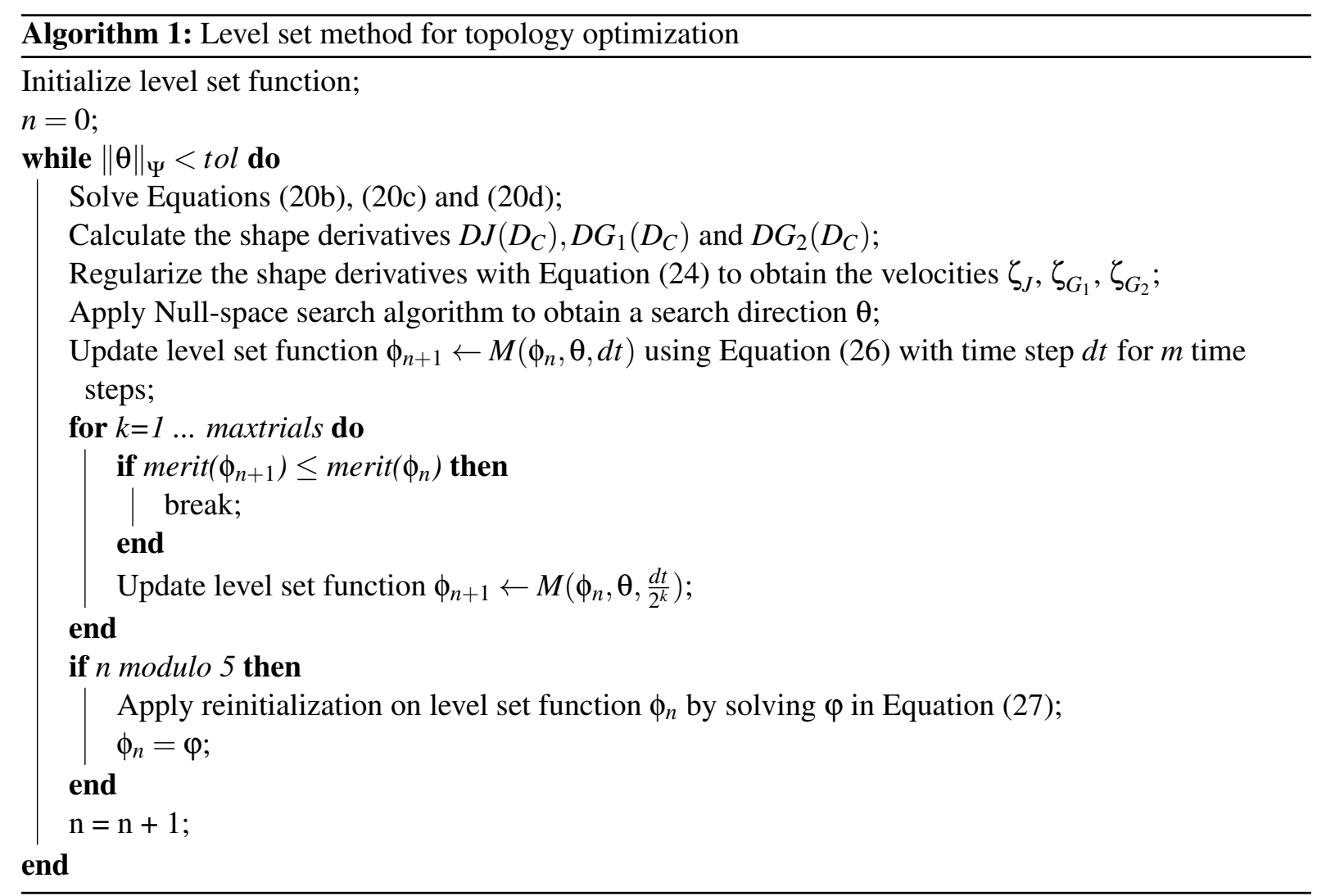

outlets $\Gamma_{C_{\text {out }}}$ and $\Gamma_{H_{\text {out }}}$ respectively. For the cold fluid Stokes equation, the Dirichlet condition $\mathbf{u}_{C_{\text {in }}}$ at the inlet $\Gamma_{C_{\text {in }}}$ is a horizontal parabolic profile with a maximum velocity $V_{\max }=2 \times 10^{-3} \mathrm{~m} / \mathrm{s}$. At the outlet $\Gamma_{C_{\text {out }}}$, zero pressure is imposed. The remaining boundary $\Gamma \backslash\left(\Gamma_{C_{\text {in }}} \cup \Gamma_{C_{\text {out }}}\right)$ has no-slip condition $\mathbf{u}_{C}=0$. The hot fluid Stokes equation has the same boundary conditions, but on $\Gamma_{H_{\text {in }}}, \Gamma_{H_{\text {out }}}$ and $\Gamma \backslash\left(\Gamma_{H_{\text {in }}} \cup \Gamma_{H_{\text {out }}}\right)$ respectively. For the temperature field, the Dirichlet conditions are $\hat{T}_{C}=10$ on $\Gamma_{C_{\text {in }}}$ and $\hat{T}_{H}=100$ on $\Gamma_{H_{\text {in }}}$. The specific heat capacity is $c_{p}=5 \times 10^{5} \mathrm{~J} /\left(\mathrm{kg}{ }^{\circ} \mathrm{C}\right)$; the density $\rho, 1 \mathrm{~kg} / \mathrm{m}^{3}$, and the conductivity, $k=1$ Watts $/\left(\mathrm{m}^{\circ} \mathrm{C}\right)$. The non-design domain $D \backslash D_{D}$ are the colored regions. The hot (red) fixed region is treated as solid material in the cold fluid analysis while the cold (blue) fixed region is treated as solid material in the hot fluid analysis. The reason to exclude these colored regions from the design domain is to prevent the optimization algorithm from placing material at these locations in an attempt to increase the heat transfer, but at the expense of blocking the flow. The value for the Brinkman penalty term $\alpha$ is $\frac{5}{6} \times 10^{4} 1 / \mathrm{s}$. We found this value to return the most reasonable results. Even though higher values of $\alpha$ approximate the solid domain better, we observed that the optimization algorithm converges to a poorer design minimum. We decided not to pursue a continuation strategy to increase $\alpha$, but it will be considered in future work. The mesh is created with Gmsh [52]. We provide a python script to generate the Gmsh file using pygmsh [77].

The Stokes-Brinkman and heat transfer equations for both the volume fraction, cf. Equations (18b , $18 \mathrm{c}$ and 18d) and level set methods, cf. Equation (20b, 20c and 20d) are implemented in Firedrake $[75,67,57]$, which uses PETSc $[15,14,34]$ as the backend for the linear algebra. Due to the small size 


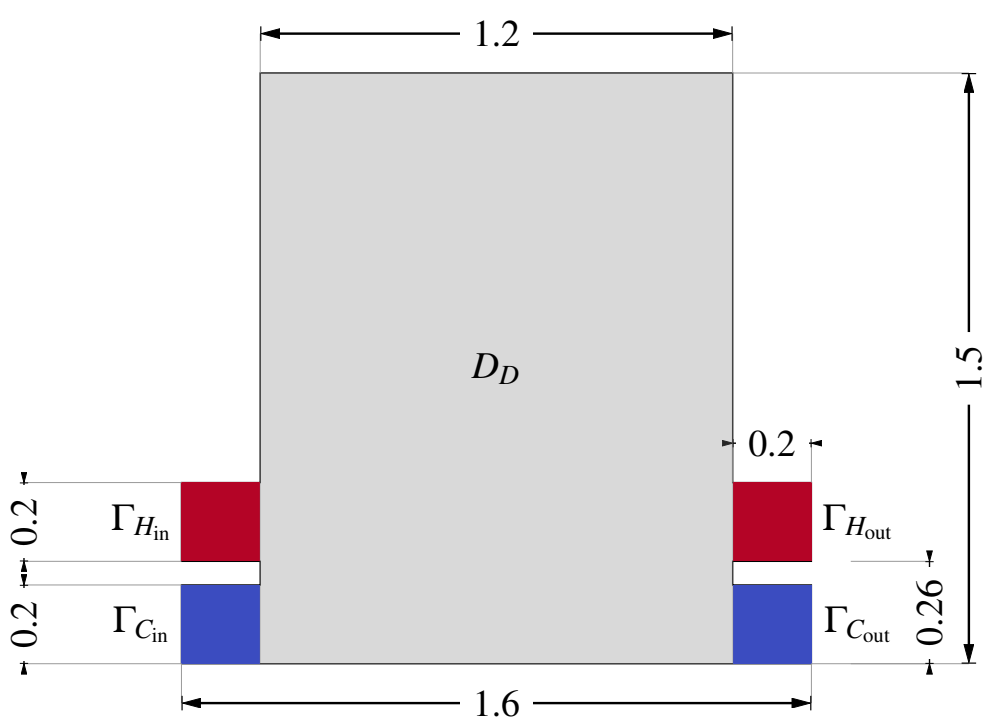

Figure 1: Simulation domain. All dimensions are in meters.

of the problem, we use the direct solver MUMPS $[9,10]$. The simulations were run on a single $2.60 \mathrm{GHz}$ Intel XeonE5-2670 processor.

In the volume fraction optimization, we start with a uniform initial value of $v_{C}=0.5$. The derivatives are calculated automatically with pyadjoint [68] and we use MMA [80] as the optimization algorithm. During the optimization, we use $\left\|v_{C}^{n+1}-v_{C}^{n}\right\|_{\infty} \leq t o l$ as termination criteria. To obtain better discrete designs, we use a continuation strategy on the SIMP coefficient $p_{\text {SIMP }}$. Starting from a value of $p_{\text {SIMP }}=5$, the optimization proceeds until the termination criteria is below $10^{-4}$ or $10^{-2}$ for more than ten iterations. We then decrease $p_{\text {SIMP }}$ in Equation (17) to 3 so that intermediate densities impose greater penalization on the pressure drop and the optimized design is crisper. Afterwards, the optimization continues until the termination criteria is below $10^{-8}$ or $10^{-3}$ for more than ten iterations, or a total simulation time of twenty hours has passed. This sequence of $p_{\text {SIMP }}$ values is opposite to traditional topology optimization compliance problems. Nevertheless, decreasing $p_{\text {SIMP }}$ from 5 to 3 generated much better designs than increasing it from 3 to 5 .

We plot the results in Table 1 for varying viscosity values of $\mu=0.01,0.02,0.04$ and $0.08 \mathrm{~m}^{2} / \mathrm{s}$. All designs feature similar tortuous flow paths as seen in [71] to maximize the interface area between both flows. For the same design, a lower viscosity translates into a lower pressure drop. Therefore, the optimized designs have more narrow channels with more interface area between the fluid channels and hence greater heat transfer. A greater interface area also implies a greater design complexity, and perhaps this is why the optimization algorithm takes more design iterations, cf. Figure 2 . The optimized design for $\mu=0.01 \mathrm{~m}^{2} / \mathrm{s}$ shows very thin fins due to a lack of control on the minimum length scale. Indeed, the pressure constraint only prevents narrow flow cross sections, but cannot prevent thin fins from forming. We intentionally did not to use any filtering length scale control techniques commonly used in volume fraction based topology optimization $[65,23,26]$ to constrain the widths of these fins. These filtering methods increase the size of the interphase regions. We will investigate other methods to constrain the 
minimum length scale while controlling the extent of the interphase region. Examples of such techniques are projection methods [85] or higher order parametrizations of the volume fraction such as B-splines [74] or geometry projection methods [69].

Figure 2 shows the cost function histories in the optimization for $\mu=0.01,0.02,0.04$ and $0.08 \mathrm{~m}^{2} / \mathrm{s}$. The discontinuity is due to the change in the SIMP exponent in Equation (17) from 5 to 3.

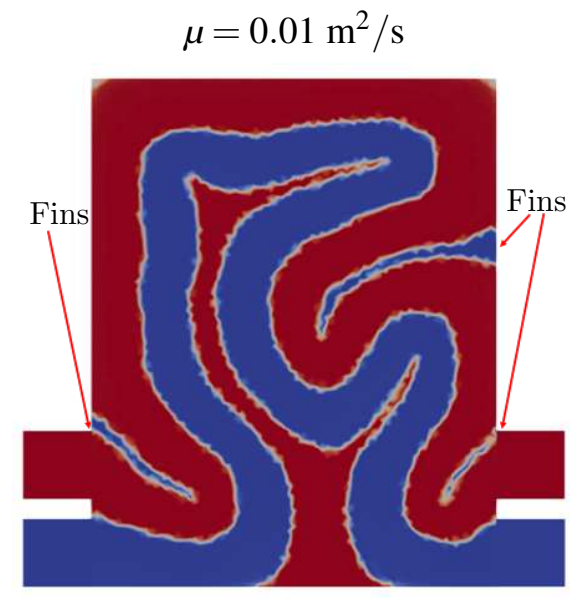

$$
\mu=0.04 \mathrm{~m}^{2} / \mathrm{s}
$$
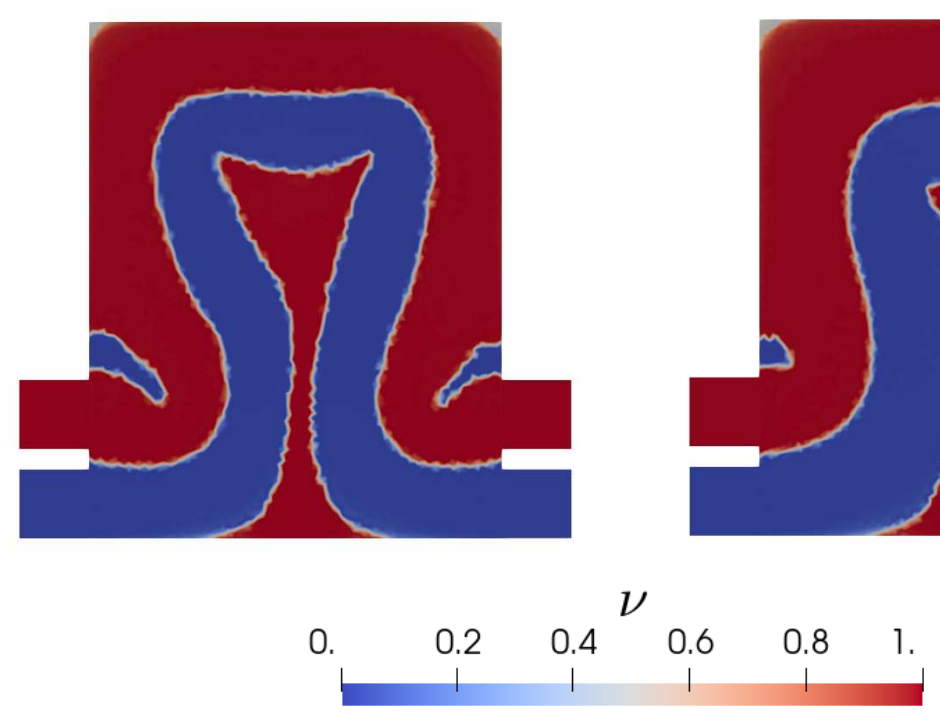

$$
\mu=0.02 \mathrm{~m}^{2} / \mathrm{s}
$$

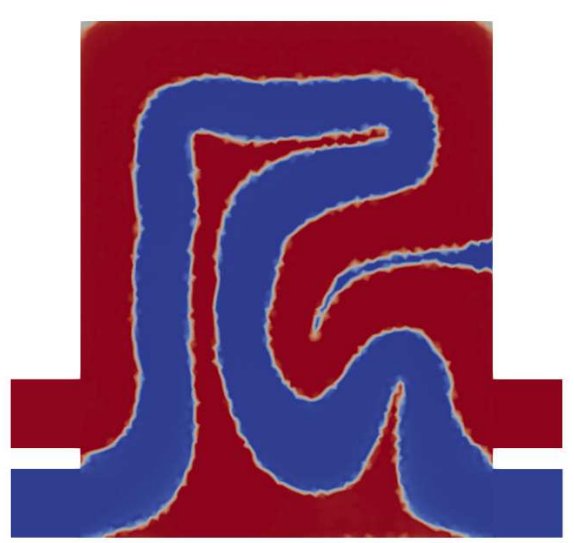

$$
\mu=0.08 \mathrm{~m}^{2} / \mathrm{s}
$$

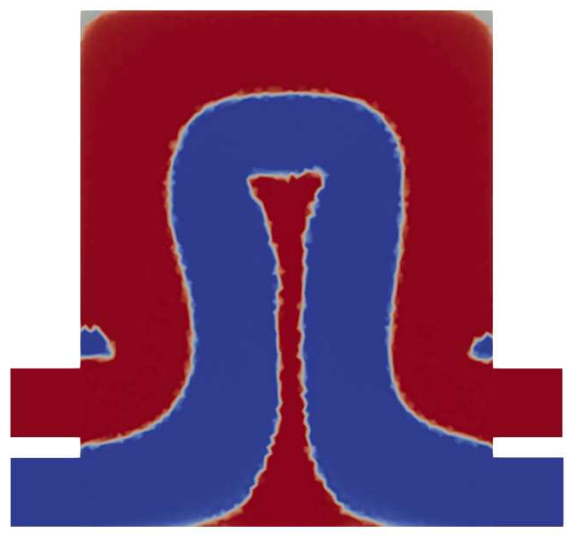

\section{(1)}

Table 1: Optimized designs for the heat exchanger using the volume fraction approach.

For the optimized design with $\mu=0.02 \mathrm{~m}^{2} / \mathrm{s}$, we plot the temperature field in Figure 3 and the velocity magnitude for both fluids in Figure 4. In Figure 3, the temperature gradient across the interface separating the fluids is greater at the inlet region, and continually decreases towards the outlet region due to fact that the hot(cold) fluid becomes colder(hotter) downstream from the inlet and thus the temperature difference across the interface decreases downstream, as expected. The velocity magnitude in both fluids, cf. Figure 


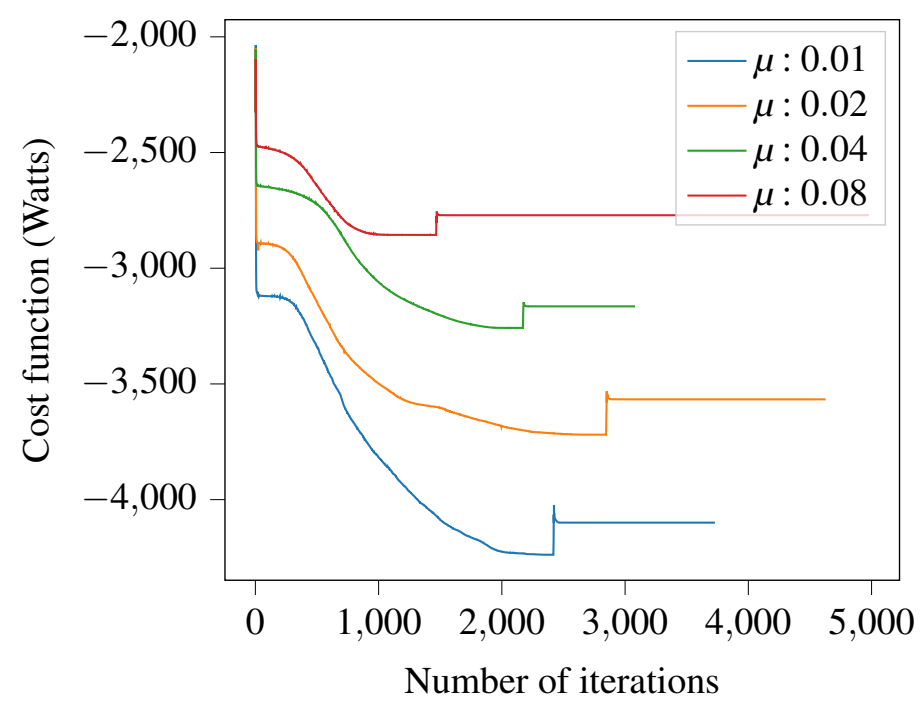

Figure 2: Optimization histories for viscosity values $\mu=0.08,0.04,0.02,0.01 \mathrm{~m}^{2} / \mathrm{s}$ when using the volume fraction method.

\begin{tabular}{rr} 
Viscosity $\mu\left(\mathrm{m}^{2} / \mathrm{s}\right)$ & Cost function (Watts) \\
\hline 0.08 & 2777.48 \\
0.04 & 3177.52 \\
0.02 & 3596.67 \\
0.01 & 4082.71
\end{tabular}

Table 2: Cost function values using the volume fraction method.

4 shows a fairly uniform profile along the entire flow paths.

We solve the same problem using the level set method. Our initial design is always $\phi(x, y)=\sin \left(\frac{y \pi}{5}\right) \cos \left(\frac{x \pi}{5}\right)-$ $\frac{4}{5}$, cf. Figure 5 as is the $\gamma=1$ regularization parameter. The scalar field $\phi: D \rightarrow \mathbb{R}$ and the velocity fields $\zeta_{J}, \zeta_{G_{1}}$ and $\zeta_{G_{2}}$ components are discretized with first order Lagrange elements on the same mesh that is used to discretize the Stokes-Brinkman equations (7). We plot the final designs in Table 3 and denote the values of the cost function in Table 4 . The results are similar to those obtained by the volume fraction method, i.e. higher complexity for lower viscosity $\mu$ values.

A one-to-one comparison between both methods is not rigorous because different optimization algorithms were employed. However, it is worthwhile to highlight the differences between them. Namely, the level set method requires fewer iterations to converge, at the expense of two shortcomings: First, the level set method is not able to nucleate new holes in the domain, it is only capable of merging existing ones. In the case of the heat exchanger, the level set method is not capable of rounding the upper left and right corners to decrease the pressure drop in the hot fluid. This inability to create new holes has been addressed for elasticity problems using topological derivatives $[49,11]$ to determine the optimal location of the holes during the optimization [5, 28] or via a complementary volume-fraction/density field [16]. Secondly, the lack of a mechanism to control the minimum length scale affects the convergence of the level set method for $\mu=0.01$ and $\mu=0.02 \mathrm{~m}^{2} / \mathrm{s}$, cf. Figure 6 . We observe the optimization algorithm 


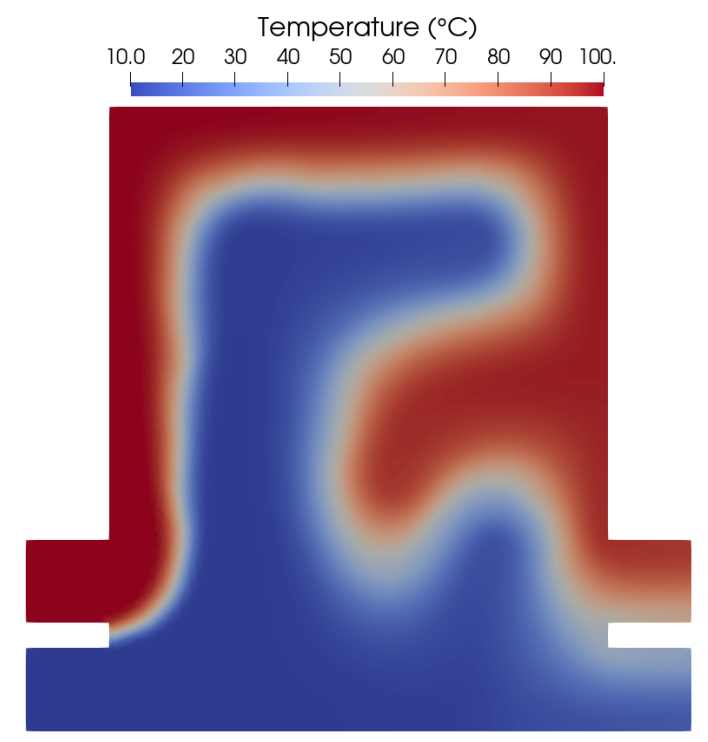

Figure 3: Temperature field for the optimized design with $\mu=0.02 \mathrm{~m}^{2} / \mathrm{s}$.

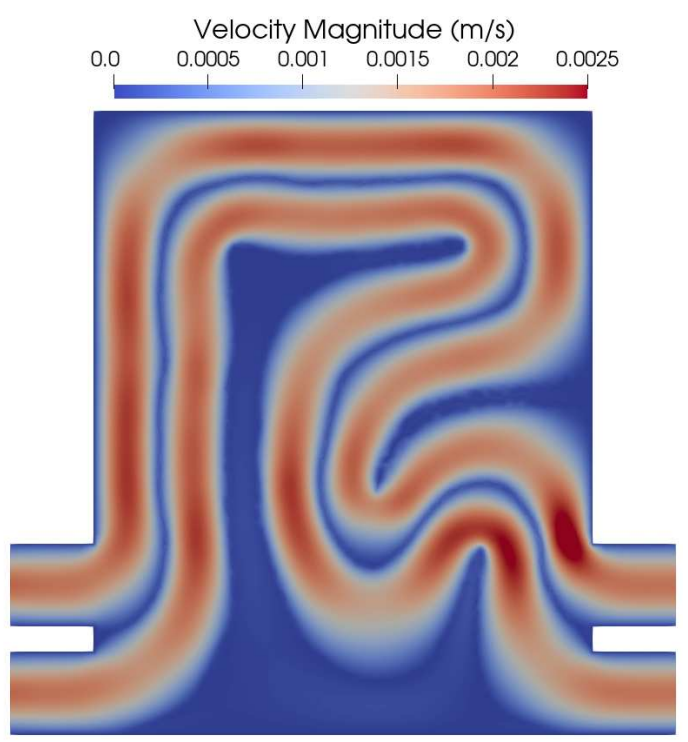

Figure 4: Velocity magnitude for cold and hot fluids for the optimized design with $\mu=0.02 \mathrm{~m}^{2} / \mathrm{s}$.

struggling as it is incapable of creating thinner features, as evidenced by multiple failed line searches. Several methods address this issue by exploiting the geometric information given by the signed-distance function. For instance, length-scale constraints can be formulated by monitoring the distance from the boundary to the skeleton of the structure $[6,88,53]$ or by comparing the areas enclosed by a boundary offset and by a level set offset [87]. Approaches that parametrize the level set with an explicit representation utilize the length-scale constraints introduced in structural topology optimization methods $[59,43]$. Another contributor to the erratic convergence is attributed to the current linear interpolation of 


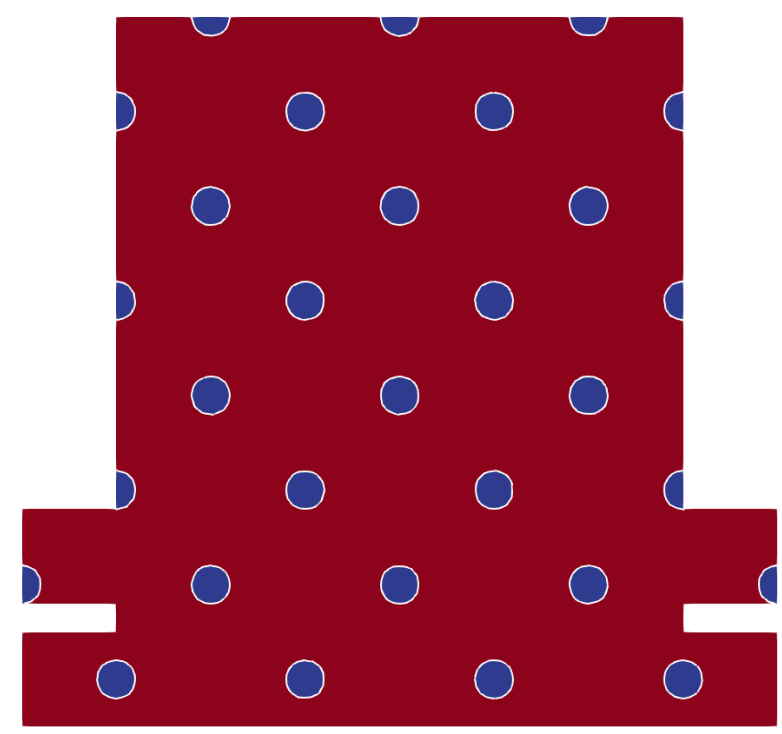

Figure 5: Initial design.

the heaviside function $H \circ \phi$ in elements where $\phi$ changes sign. Indeed, this mapping creates an irregular interface that can cause undesirable oscillations in the shape derivatives. This is specially noticeable when a geometry feature is not sufficiently refined. For this reason, in our future work, we will consider the implementation of an immersed type finite element method such as CutFEM [29] or XFEM [17].

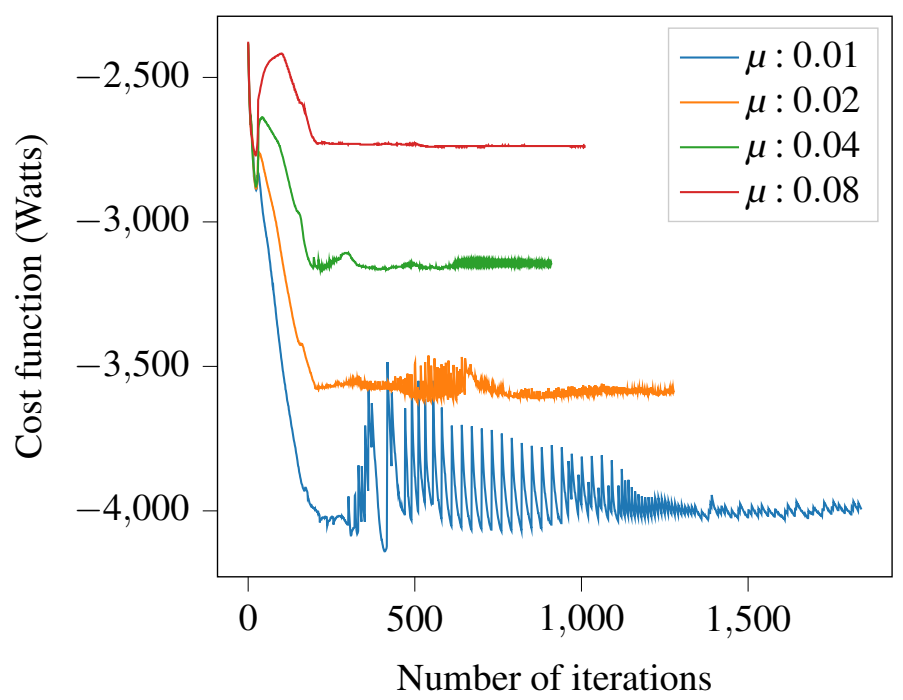

Figure 6: Optimization histories for viscosity values $\mu=0.08,0.04,0.02,0.01 \mathrm{~m}^{2} / \mathrm{s}$ when using the level set method. 

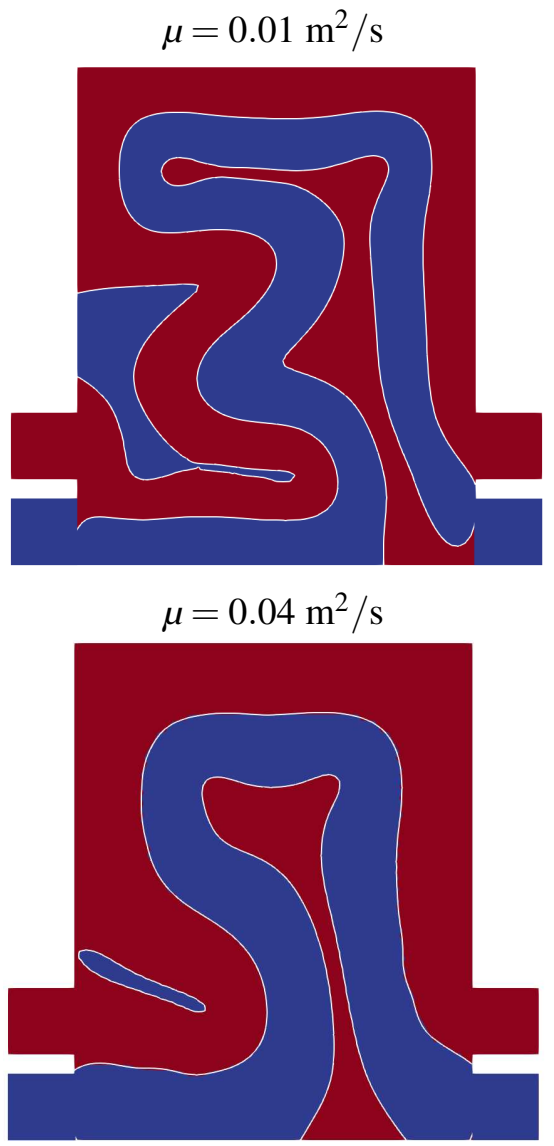

$H \circ \phi$

0

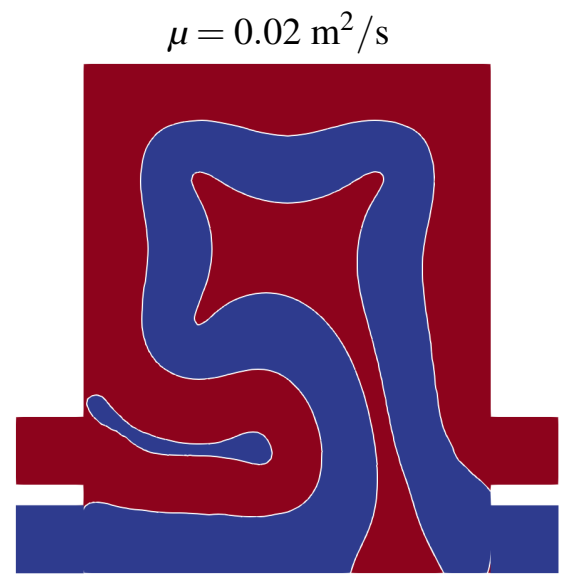

$\mu=0.08 \mathrm{~m}^{2} / \mathrm{s}$

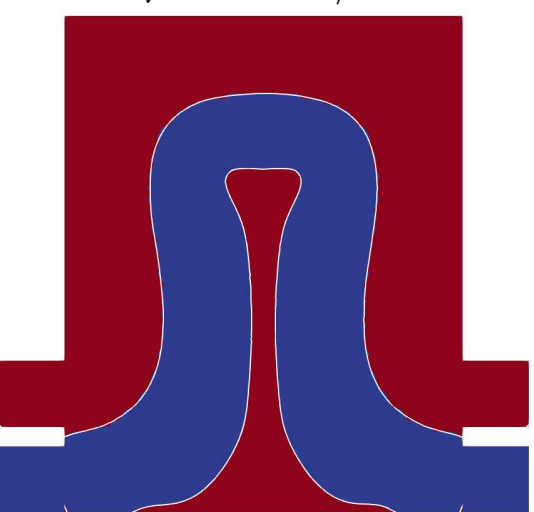

1.

Table 3: Optimized designs for the heat exchanger using the level set approach. The designs have been post processed with Paraview [13] to obtain the interface defined by $\phi(\mathbf{x})=0$.

\begin{tabular}{rr} 
Viscosity $\mu\left(\mathrm{m}^{2} / \mathrm{s}\right)$ & Cost function (Watts) \\
\hline 0.08 & 2737.51 \\
0.04 & 3155.25 \\
0.02 & 3587.45 \\
0.01 & 4007.62
\end{tabular}

Table 4: Cost function values for the examples using the level set method.

\section{Conclusions}

We present the two dimensional topology optimizations of a two fluid heat exchanger using the volume fraction and level set methods. To prevent both fluids from mixing, we solve the Stokes equation for each fluid with a Brinkman penalization modeling the other phase as solid. The velocity fields from 
both fluids are then fed into the heat transfer equation as the advection field to obtain the temperature. Using these governing equations, we proceed to maximize the heat transfer between both fluids with a constraint on the pressure drop across each fluid channel. The volume fraction method uses the SIMP penalization and the level set method reformulates the problem as a shape optimization with the domains defined by a level set. We solve the same optimization problem for several values of the viscosity using both methods and compare the results. The optimized designs obtained share similar geometry and heat transfer efficiencies. However, the level set method converges in fewer design of iterations at the expense of heavy oscillations for lower viscosity values due to a lack of minimum length control.

The most immediate improvement for the presented framework is the enforcement of a length scale control method for the level set method. Such methods have already been proposed in the literature $[6,87]$ and their implementations are not difficult. Our plan is to also perform optimizations for three dimensional domains, where more interesting designs can be obtained. Likewise, in three dimensions it will be worth investigating different flow configurations, i.e. a comparison between parallel, cross flow, shell and tube heat exchangers, etc. Lastly, we have assumed an infinitesimally thin membrane separating both fluids. A wall with non-zero thickness and different conductivity properties will offer more realistic designs.

\section{Replication of results}

The code to replicate the volume fraction results is in the repository https://github.com/LLNL/ 2DHEVE and it requires the installation of the MMA python implementation found in https://github. com/LLNL/pyMMAopt and Signac (https://signac.io/) to run the parameter sweep. The level set method results can be replicated with the library lestofire https://github.com/LLNL/lestofire, which contains the heat exchanger design in one of the examples, i.e. https://github.com/LLNL/ lestofire/tree/main/examples/heat_exchanger. Both repositories require the Firedrake library (https://www.firedrakeproject.org/).

\section{Conflict of interest.}

The authors declare that they have no conflict of interest.

\section{Funding information}

This work was performed under the auspices of the U.S. Department of Energy by Lawrence Livermore National Laboratory under Contract DE-AC52-07NA27344. LLNL-JRNL-816310.

\section{REFERENCES}

[1] J. Alexandersen, N. Aage, C. S. Andreasen, and O. Sigmund. Topology optimisation for natural convection problems. International Journal for Numerical Methods in Fluids, 76(10):699-721, 2014.

[2] J. Alexandersen and C. S. Andreasen. A review of topology optimisation for fluid-based problems. Fluids, 5(1):29, 2020.

[3] G. Allaire, C. Dapogny, G. Delgado, and G. Michailidis. Multi-phase structural optimization via a level set method. ESAIM: control, optimisation and calculus of variations, 20(2):576-611, 2014. 
[4] G. Allaire, C. Dapogny, and P. Frey. Topology and geometry optimization of elastic structures by exact deformation of simplicial mesh. Comptes Rendus Mathematique, 349(17-18):999-1003, 2011.

[5] G. Allaire, F. De Gournay, F. Jouve, and A.-M. Toader. Structural optimization using topological and shape sensitivity via a level set method. Control and cybernetics, 34(1):59, 2005.

[6] G. Allaire, F. Jouve, and G. Michailidis. Thickness control in structural optimization via a level set method. Structural and Multidisciplinary Optimization, 53(6):1349-1382, 2016.

[7] G. Allaire, F. Jouve, and A.-M. Toader. A level-set method for shape optimization. Comptes Rendus Mathematique, 334(12):1125-1130, 2002.

[8] G. Allaire, F. Jouve, and A.-M. Toader. Structural optimization using sensitivity analysis and a level-set method. Journal of computational physics, 194(1):363-393, 2004.

[9] P. R. Amestoy, I. S. Duff, J.-Y. L'Excellent, and J. Koster. A fully asynchronous multifrontal solver using distributed dynamic scheduling. SIAM Journal on Matrix Analysis and Applications, 23(1):15-41, 2001.

[10] P. R. Amestoy, A. Guermouche, J.-Y. L'Excellent, and S. Pralet. Hybrid scheduling for the parallel solution of linear systems. Parallel Computing, 32(2):136-156, 2006.

[11] S. Amstutz and H. Andrä. A new algorithm for topology optimization using a level-set method. Journal of computational physics, 216(2):573-588, 2006.

[12] M. Awais and A. A. Bhuiyan. Heat and mass transfer for compact heat exchanger (chxs) design: A state-of-the-art review. International Journal of Heat and Mass Transfer, 127:359-380, 2018.

[13] U. Ayachit. The paraview guide: a parallel visualization application. 2015.

[14] S. Balay, S. Abhyankar, M. F. Adams, J. Brown, P. Brune, K. Buschelman, L. Dalcin, V. Eijkhout, W. D. Gropp, D. Karpeyev, D. Kaushik, M. G. Knepley, D. A. May, L. C. McInnes, R. T. Mills, T. Munson, K. Rupp, P. Sanan, B. F. Smith, S. Zampini, H. Zhang, and H. Zhang. PETSc users manual. Technical Report ANL-95/11 - Revision 3.11, Argonne National Laboratory, 2019.

[15] S. Balay, W. D. Gropp, L. C. McInnes, and B. F. Smith. Efficient management of parallelism in object oriented numerical software libraries. In E. Arge, A. M. Bruaset, and H. P. Langtangen, editors, Modern Software Tools in Scientific Computing, pages 163-202. Birkhäuser Press, 1997.

[16] J. L. Barrera, M. J. Geiss, and K. Maute. Hole seeding in level set topology optimization via density fields. Structural and Multidisciplinary Optimization, pages 1-25, 2020.

[17] T. Belytschko, C. Parimi, N. Moës, N. Sukumar, and S. Usui. Structured extended finite element methods for solids defined by implicit surfaces. International journal for numerical methods in engineering, 56(4):609-635, 2003.

[18] T. Belytschko, S. Xiao, and C. Parimi. Topology optimization with implicit functions and regularization. International Journal for Numerical Methods in Engineering, 57(8):1177-1196, 2003.

[19] M. P. Bendsøe. Optimal shape design as a material distribution problem. Structural optimization, 1(4):193-202, 1989.

[20] M. P. Bendsøe and O. Sigmund. Material interpolation schemes in topology optimization. Archive 
of applied mechanics, 69(9-10):635-654, 1999.

[21] M. Berggren. A unified discrete-continuous sensitivity analysis method for shape optimization. In Applied and numerical partial differential equations, pages 25-39. Springer, 2010.

[22] T. Borrvall and J. Petersson. Topology optimization of fluids in stokes flow. International journal for numerical methods in fluids, 41(1):77-107, 2003.

[23] B. Bourdin. Filters in topology optimization. International journal for numerical methods in engineering, 50(9):2143-2158, 2001.

[24] F. Brezzi. On the existence, uniqueness and approximation of saddle-point problems arising from lagrangian multipliers. Publications mathématiques et informatique de Rennes, (S4):1-26, 1974.

[25] A. N. Brooks and T. J. Hughes. Streamline upwind/petrov-galerkin formulations for convection dominated flows with particular emphasis on the incompressible navier-stokes equations. Computer methods in applied mechanics and engineering, 32(1-3):199-259, 1982.

[26] T. E. Bruns and D. A. Tortorelli. Topology optimization of non-linear elastic structures and compliant mechanisms. Computer methods in applied mechanics and engineering, 190(26-27):34433459, 2001.

[27] M. Burger. A framework for the construction of level set methods for shape optimization and reconstruction. Interfaces and Free boundaries, 5(3):301-329, 2003.

[28] M. Burger, B. Hackl, and W. Ring. Incorporating topological derivatives into level set methods. Journal of Computational Physics, 194(1):344-362, 2004.

[29] E. Burman, S. Claus, P. Hansbo, M. G. Larson, and A. Massing. Cutfem: discretizing geometry and partial differential equations. International Journal for Numerical Methods in Engineering, 104(7):472-501, 2015.

[30] E. Burman, D. Elfverson, P. Hansbo, M. G. Larson, and K. Larsson. A cut finite element method for the bernoulli free boundary value problem. Computer Methods in Applied Mechanics and Engineering, 317:598-618, 2017.

[31] E. Burman, D. Elfverson, P. Hansbo, M. G. Larson, and K. Larsson. Shape optimization using the cut finite element method. Computer Methods in Applied Mechanics and Engineering, 328:242$261,2018$.

[32] E. Burman and P. Hansbo. Edge stabilization for galerkin approximations of convection-diffusionreaction problems. Computer methods in applied mechanics and engineering, 193(15-16):14371453, 2004.

[33] K. K. Choi and H. G. Seong. Design component method for sensitivity analysis of built-up structures. Journal of structural mechanics, 14(3):379-399, 1986.

[34] L. D. Dalcin, R. R. Paz, P. A. Kler, and A. Cosimo. Parallel distributed computing using Python. Advances in Water Resources, 34(9):1124-1139, 2011. New Computational Methods and Software Tools.

[35] T. Dbouk. A review about the engineering design of optimal heat transfer systems using topology optimization. Applied Thermal Engineering, 112:841-854, 2017. 
[36] F. De Gournay. Velocity extension for the level-set method and multiple eigenvalues in shape optimization. SIAM journal on control and optimization, 45(1):343-367, 2006.

[37] J. D. Deaton and R. V. Grandhi. A survey of structural and multidisciplinary continuum topology optimization: post 2000. Structural and Multidisciplinary Optimization, 49(1):1-38, 2014.

[38] E. M. Dede. Multiphysics topology optimization of heat transfer and fluid flow systems. In In Proceedings of the COMSOL Conference 2009, , Boston, MA, USA, 8-10 October 2009., 2009.

[39] M. C. Delfour and J.-P. Zolésio. Shapes and geometries: metrics, analysis, differential calculus, and optimization, volume 22. Siam, 2011.

[40] T. Dixit and I. Ghosh. Review of micro-and mini-channel heat sinks and heat exchangers for single phase fluids. Renewable and Sustainable Energy Reviews, 41:1298-1311, 2015.

[41] J. S. Dokken, S. K. Mitusch, and S. W. Funke. Automatic shape derivatives for transient pdes in fenics and firedrake. arXiv preprint arXiv:2001.10058, 2020.

[42] P. Dular, C. Geuzaine, F. Henrotte, and W. Legros. A general environment for the treatment of discrete problems and its application to the finite element method. IEEE Transactions on Magnetics, 34(5):3395-3398, 1998.

[43] P. D. Dunning. Minimum length-scale constraints for parameterized implicit function based topology optimization. Structural and multidisciplinary optimization, 58(1):155-169, 2018.

[44] P. D. Dunning and H. A. Kim. Introducing the sequential linear programming level-set method for topology optimization. Structural and Multidisciplinary Optimization, 51(3):631-643, 2015.

[45] F. Feppon. Optimisation topologique de systèmes multiphysiques. Theses, Université Paris-Saclay, Dec. 2019.

[46] F. Feppon. Null space optimizer, 2020. https://pypi.org/project/nullspace-optimizer/.

[47] F. Feppon, G. Allaire, and C. Dapogny. Null space gradient flows for constrained optimization with applications to shape optimization. working paper or preprint, Jan. 2019.

[48] K. Foli, T. Okabe, M. Olhofer, Y. Jin, and B. Sendhoff. Optimization of micro heat exchanger: Cfd, analytical approach and multi-objective evolutionary algorithms. International Journal of Heat and Mass Transfer, 49(5-6):1090-1099, 2006.

[49] S. Garreau, P. Guillaume, and M. Masmoudi. The topological asymptotic for pde systems: the elasticity case. SIAM journal on control and optimization, 39(6):1756-1778, 2001.

[50] A. Gersborg-Hansen, M. P. Bendsøe, and O. Sigmund. Topology optimization of heat conduction problems using the finite volume method. Structural and multidisciplinary optimization, 31(4):251-259, 2006.

[51] A. Gersborg-Hansen, O. Sigmund, and R. B. Haber. Topology optimization of channel flow problems. Structural and Multidisciplinary Optimization, 30(3):181-192, 2005.

[52] C. Geuzaine and J.-F. Remacle. Gmsh: A 3-d finite element mesh generator with built-in pre-and post-processing facilities. International journal for numerical methods in engineering, 79(11):1309-1331, 2009. 
[53] X. Guo, W. Zhang, and W. Zhong. Explicit feature control in structural topology optimization via level set method. Computer Methods in Applied Mechanics and Engineering, 272:354-378, 2014.

[54] J. H. Haertel and G. F. Nellis. A fully developed flow thermofluid model for topology optimization of 3d-printed air-cooled heat exchangers. Applied thermal engineering, 119:10-24, 2017.

[55] D. A. Ham, L. Mitchell, A. Paganini, and F. Wechsung. Automated shape differentiation in the unified form language. Structural and Multidisciplinary Optimization, 60(5):1813-1820, 2019.

[56] R. Hiptmair, A. Paganini, and S. Sargheini. Comparison of approximate shape gradients. BIT Numerical Mathematics, 55(2):459-485, 2015.

[57] M. Homolya, L. Mitchell, F. Luporini, and D. A. Ham. TSFC: a structure-preserving form compiler, 2017.

[58] L. C. Høghøj, D. R. Nørhave, J. Alexandersen, O. Sigmund, and C. S. Andreasen. Topology optimization of two fluid heat exchangers. International Journal of Heat and Mass Transfer, 163:120543, 2020.

[59] M. Jansen. Explicit level set and density methods for topology optimization with equivalent minimum length scale constraints. Structural and Multidisciplinary Optimization, 59(5):1775-1788, 2019.

[60] G. Kiziltas, D. Psychoudakis, J. L. Volakis, and N. Kikuchi. Topology design optimization of dielectric substrates for bandwidth improvement of a patch antenna. IEEE Transactions on Antennas and Propagation, 51(10):2732-2743, 2003.

[61] H. Kobayashi, K. Yaji, S. Yamasaki, and K. Fujita. Freeform winglet design of fin-and-tube heat exchangers guided by topology optimization. Applied Thermal Engineering, 161:114020, 072019.

[62] H. Kobayashi, K. Yaji, S. Yamasaki, and K. Fujita. Topology design of two-fluid heat exchange. Structural and Multidisciplinary Optimization, Oct 2020.

[63] S. Kreissl and K. Maute. Levelset based fluid topology optimization using the extended finite element method. Structural and Multidisciplinary Optimization, 46(3):311-326, 2012.

[64] A. Laurain. A level set-based structural optimization code using fenics. Structural and Multidisciplinary Optimization, 58(3):1311-1334, 2018.

[65] B. S. Lazarov and O. Sigmund. Filters in topology optimization based on helmholtz-type differential equations. International Journal for Numerical Methods in Engineering, 86(6):765-781, 2011.

[66] Q. Li, G. P. Steven, O. M. Querin, and Y. Xie. Shape and topology design for heat conduction by evolutionary structural optimization. International Journal of Heat and Mass Transfer, 42(17):3361-3371, 1999.

[67] F. Luporini, D. A. Ham, and P. H. J. Kelly. An algorithm for the optimization of finite element integration loops. ACM Transactions on Mathematical Software, 44:3:1-3:26, 2017.

[68] S. Mitusch, S. Funke, and J. Dokken. dolfin-adjoint 2018.1: automated adjoints for fenics and firedrake. Journal of Open Source Software, 4(38):1292, 2019.

[69] J. Norato, B. Bell, and D. A. Tortorelli. A geometry projection method for continuum-based topol- 
ogy optimization with discrete elements. Computer Methods in Applied Mechanics and Engineering, 293:306-327, 2015.

[70] S. Osher and J. A. Sethian. Fronts propagating with curvature-dependent speed: algorithms based on hamilton-jacobi formulations. Journal of computational physics, 79(1):12-49, 1988.

[71] P. Panagiotis. Topology optimization of heat exchangers, 2015.

[72] V. K. Patel, V. J. Savsani, and M. A. Tawhid. Thermal Design and Optimization of Heat Exchangers, pages 33-98. Springer International Publishing, Cham, 2019.

[73] D. Peng, B. Merriman, S. Osher, H. Zhao, and M. Kang. A pde-based fast local level set method. Journal of computational physics, 155(2):410-438, 1999.

[74] X. Qian. Topology optimization in b-spline space. Computer Methods in Applied Mechanics and Engineering, 265:15-35, 2013.

[75] F. Rathgeber, D. A. Ham, L. Mitchell, M. Lange, F. Luporini, A. T. T. McRae, G.-T. Bercea, G. R. Markall, and P. H. J. Kelly. Firedrake: automating the finite element method by composing abstractions. ACM Trans. Math. Softw., 43(3):24:1-24:27, 2016.

[76] K. R. Saviers, R. Ranjan, and R. Mahmoudi. Design and validation of topology optimized heat exchangers. In AIAA Scitech 2019 Forum, page 1465, 2019.

[77] N. Schlömer, A. Cervone, G. McBain, tryfon mw, R. van Staden, F. Gokstorp, toothstone, J. S. Dokken, anzil, J. Sanchez, D. Kempf, M. Bussonnier, Y. Feng, awa5114, T. Maric, S. Chen, nilswagner, Nate, ivanmultiwave, and F. Fu. nschloe/pygmsh v6.1.1, Apr. 2020.

[78] J. Sethian and A. Wiegmann. Structural boundary design via level set and immersed interface methods. Journal of Computational Physics, 163(2):489 - 528, 2000.

[79] R. K. Shah. Advances in science and technology of compact heat exchangers. Heat Transfer Engineering, 27(5):3-22, 2006.

[80] K. Svanberg. The method of moving asymptotes-a new method for structural optimization. International journal for numerical methods in engineering, 24(2):359-373, 1987.

[81] A. D. Tharkar and S. P. Mahulikar. Size Effect on Thermal Characteristic of Tubular Heat Exchanger at Miniscale and Microscale. Journal of Thermal Science and Engineering Applications, 11(2), 10 2018. 021001.

[82] N. P. van Dijk, K. Maute, M. Langelaar, and F. Van Keulen. Level-set methods for structural topology optimization: a review. Structural and Multidisciplinary Optimization, 48(3):437-472, 2013.

[83] C. H. Villanueva and K. Maute. Cutfem topology optimization of 3d laminar incompressible flow problems. Computer Methods in Applied Mechanics and Engineering, 320:444-473, 2017.

[84] E. Wadbro and M. Berggren. Topology optimization of an acoustic horn. Computer Methods in Applied Mechanics and Engineering, 196:420-436, 122006.

[85] F. Wang, B. S. Lazarov, and O. Sigmund. On projection methods, convergence and robust formulations in topology optimization. Structural and Multidisciplinary Optimization, 43(6):767-784, 2011. 
[86] M. Y. Wang, X. Wang, and D. Guo. A level set method for structural topology optimization. Computer methods in applied mechanics and engineering, 192(1-2):227-246, 2003.

[87] Y. Wang, L. Zhang, and M. Y. Wang. Length scale control for structural optimization by level sets. Computer Methods in Applied Mechanics and Engineering, 305:891-909, 2016.

[88] Q. Xia and T. Shi. Constraints of distance from boundary to skeleton: for the control of length scale in level set based structural topology optimization. Computer Methods in Applied Mechanics and Engineering, 295:525-542, 2015.

[89] S. Yamasaki, T. Nomura, A. Kawamoto, K. Sato, and S. Nishiwaki. A level set-based topology optimization method targeting metallic waveguide design problems. International Journal for $\mathrm{Nu}$ merical Methods in Engineering, 87(9):844-868, 2011.

[90] G. H. Yoon. Topological design of heat dissipating structure with forced convective heat transfer. Journal of Mechanical Science and Technology, 24(6):1225-1233, 2010.

[91] M. Zhou and G. Rozvany. The coc algorithm, part ii: Topological, geometrical and generalized shape optimization. Computer Methods in Applied Mechanics and Engineering, 89(1):309 - 336, 1991. Second World Congress on Computational Mechanics.

[92] S. Zhou, W. Li, and Q. Li. Level-set based topology optimization for electromagnetic dipole antenna design. Journal of Computational Physics, 229(19):6915-6930, 2010.

\section{A Hamilton-Jacobi equation solver}

To stabilize the solution to the Hamilton-Jacobi Equation (26), we follow [32] and add a term penalizing he gradient jumps across elemen boundaries weighted by he stabilizaion parameter $c_{2}$. Ultimately, we find $\phi \in H^{1}(D)$ such that

$$
\int_{D} \frac{\partial \phi}{\partial t} \psi-\theta \cdot \nabla \phi \psi d V+c_{2} \sum_{F \in \mathcal{F}_{i}} \int_{F} h^{2} \llbracket \nabla \phi \cdot \mathbf{n} \rrbracket \llbracket \nabla \psi \cdot \mathbf{n} \rrbracket d A=0 \quad \forall \psi \in H^{1}(D) .
$$

In all our examples, $c_{2}=1.0$. We use linear Lagrange elements for the space discretization and the Crank-Nicholson method for the time discretization with a time step $d t$ given by

$$
d t=\frac{0.1 h_{\min }}{\max _{D}\left(\left|\theta_{x}\right|+\left|\theta_{y}\right|\right)} .
$$

where $h_{\min }$ is the diameter of the smallest element in the mesh. As described in Algorithm 1, the time step $d t$ is halved whenever the line search fails to decrease the merit function.

\section{B Signed distance equation solver}

We add an artificial term with diffusion $\kappa=6.25$ to the reinitialization Equation (27) for stabilization purposes

$$
\frac{\partial \varphi}{\partial \tau}+\frac{\phi_{0}}{\sqrt{\phi_{0}^{2}+\varepsilon\left|\nabla \phi_{0}\right|^{2}}}(1-|\nabla \varphi|)-\kappa \nabla^{2} \varphi=0,
$$


then, we transform the Equation (27) into its weak form, i.e. we find $\varphi \in H^{1}(D)$ such that

$$
\int_{D}\left(\psi \frac{\partial \phi}{\partial \tau}+\psi \frac{\phi_{0}}{\sqrt{\phi_{0}^{2}+\varepsilon|\nabla \phi|^{2}}}(1-|\nabla \phi|)-\kappa \nabla \phi \cdot \nabla \psi\right) d V=0 \forall \psi \in H^{1}(D) .
$$

The space is discretized with linear Lagrange finite elements and in time with the Forward Euler method with a time step $d \tau=10^{-7}$ in our examples. 